\title{
Numerical simulations of effects of multiple neurotransmission on intestinal propulsion of a non-deformable bolus
}

\author{
R. MIFTAHOF†* and N. AKHMADEEV \\ $\dagger$ I-BIO Program, Pohang University of Science and Technology, Pohang 790-784, South Korea \\ $\$$ Kazan Medical University, Tatarstan, Russia \\ (Received 4 April 2006; revised 31 October 2006; in final form 15 December 2006)
}

\begin{abstract}
Electrophysiological mechanisms of co-transmission by serotonin (5-HT) and acetylcholine (ACh), co-expression of receptor types 5-HT type 3 and 4, nicotinic cholinerginc (nACh) and muscarinic cholinergic ( $\mu \mathrm{ACh}$ ), and effects of selective and non-selective $5-\mathrm{HT}_{3}$ and $5-\mathrm{HT}_{4}$ receptor agonists/antagonists, on intestinal propulsion of a solid non-deformable bolus were studied numerically. Results indicated that the propagation of the wave of excitation in the presence of $5-\mathrm{HT}_{3}$ receptor antagonists was supported by co-release of ACh. Co-stimulation of $5-\mathrm{HT}_{3}, \mathrm{nACh}$ and $\mu \mathrm{ACh}$ receptors significantly impairs propulsive activity of the gut. In an ACh-free environment, Lotronex increased the transit time of the bolus along a segment of the gut. In the presence of $\mathrm{ACh}$, Lotronex produced intensive tonic-type contractions in the longitudinal and circular smooth muscle layers and eliminated propulsive activity. Zelnorm preserved the reciprocal electromechanical relationships between the longitudinal and circular smooth muscle layers. The drug changed the normal mixing pattern of activity to an expulsive type. Treatment of the gut with selective $5 \mathrm{HT}_{4}$ receptor antagonists increased the transit time by disrupting the migrating myoelectrical complex. Cisapride increased the excitability of the myenteric nervous plexus and increased the frequency of slow waves. In the presence of Cisapride smooth muscle syncytia responded with the generation of tonic contractions, resulting in a "squeezing" type of bolus movement. Comparison of the theoretical results to in vivo and in vitro experimental data indicated satisfactory qualitative and quantitative agreement.
\end{abstract}

Keywords: Gut; Mathematical model; Co-transmission by acetylcholine and serotonin; Solid non-deformable bolus; Propulsion

\section{Introduction}

Mathematical modeling and computer simulation are universal approximators that accurately simulate complex biological phenomena comprising multiple non-linear interactions. They have become an indispensable supplement to experimental in vivo and in vitro techniques that explore inaccessible biological processes and thereby assist researchers in drug design, development and screening for potential side effects. With the latest advancement in system computational biology and the development of sophisticated mathematical models of

*Corresponding author. Email: aincompany@hotmail.com

Computational and Mathematical Methods in Medicine 
the organs of the gastrointestinal tract it has become possible to deduce intricate neuropharmacological dysfunctional mechanisms and to rationalize the development of effective therapeutic agents to treat them [1-3].

There is considerable interest in the electro-pharmacological role of acetylcholine (ACh) and serotonin (5-HT) in the pathogenesis of irritable bowel syndrome. However, understanding of the complex mechanisms involved in intricate processes responsible for the biological manifestations of the disease, i.e. visceral hypersensitivity, constipation/ diarrhea, bloating, and abdominal pain remains unsatisfactory. Significant current issues are: (i) the processes of co-transmission by multiple neurotransmitters and co-activation of different receptor types cannot be studied effectively by any of the existing experimental techniques, and (ii) traditional in vivo and in vitro approaches have failed, so far, to provide required quantitative information about the role of synaptic neurotransmission in the pathogenesis of the syndrome.

The aim of this study is to gain insight into phenomenological mechanisms of cotransmission by ACh and 5-HT and their effects on the motor and propulsive activity of the gut. This paper focuses primarily on the analysis of: (i) the concurrent effects of co-activation of muscarinic ( $\mu \mathrm{ACh})$, nicotinic (nACh) and 5-HT types 3 and 4 receptors, on the dynamics of signal transduction and electromechanical responses in the gut, and (ii) the role of pharmacological agents with selective, nonselective and mixed $5-\mathrm{HT}_{3}$ and $5-\mathrm{HT}_{4}$ receptor agonist/antagonist mechanisms of action on its propulsive activity. This is, to our knowledge, the first application of a numerical model to the study of processes of multiple neurotransmission and receptor polymodality in the gastrointestinal tract.

\section{Physiology of neurotransmission}

The ubiquitous biogenic amine, 5-hydroxytryptamine (5-HT, serotonin), is present in the neurons of the enteric nervous system, largely in enterochromaffin (EC) cells. Serotonin acts as a neurotransmitter and a paracrine messenger in the gastrointestinal tract to mediate a wide range of physiological functions [4-8]. These effects are achieved through activation of $5-\mathrm{HT}_{1}-5-\mathrm{HT}_{7}$ receptors. A distinct neural receptor, 5- $\mathrm{HT}_{3}$, belongs to the family of ligandgated ion channels. Serotonin applied by ionophoresis to a neuron with $5-\mathrm{HT}_{3}$ receptors causes a short latency $(<100 \mathrm{~ms})$ and a duration $(<2 \mathrm{~s})$ depolarization by invoking a fast inward excitation current. The latter is due to an increase in permeability of calcium $\left(g_{\mathrm{Ca} 2+}\right)$, potassium $\left(g_{\mathrm{K}+}\right)$ and sodium $\left(g_{\mathrm{Na}+}\right)$ channels [6,7]. The 5-HT 4 receptors belong to a Gos-protein-coupled family. They are positively linked to adenylyl cyclase in the second messenger signal transduction mechanism [8]. The biological effect of their pharmacological activation is correlated with an increase in permeability of calcium-activated potassium $\left(g_{\mathrm{Ca} 2+-\mathrm{K}+}\right)$ and sodium $\left(g_{\mathrm{Na}+}\right)$ channels and a decrease in permeability of potassium $\left(g_{\mathrm{K}+}\right)$ channels. The 5- $\mathrm{HT}_{3}$ and $5-\mathrm{HT}_{4}$ receptors, and $\mathrm{ACh}$-receptors are expressed on longitudinal and circular smooth muscle cells as well as the somas and presynaptic nerve terminals of the primary sensory (AH-type), motor (S-type), and possibly interneurons (S and AH-types) of the myenteric nervous plexus [9]. Electrochemical coupling at the neuro-neuronal and neuro-muscular synapses entails the initiation of cascade reactions of the second messenger system and activation of ligand-operated channels. Differences in dynamics of the implicated biochemical pathways, complex allosteric neurotransmitterreceptor interactions, and species and regional organ dependence, result in highly variable biological responses. Thus, indefinite responses (an increase or failure to affect 
gastrointestinal propulsion in the ileum of mice with an invariable inhibition of the lower intestinal motor activity) are observed after the selective blockade of $5-\mathrm{HT}_{3}-$ receptors by Ondansetron (GSK) and Lotronex (GSK). The selective stimulation of 5- $\mathrm{HT}_{4}$ receptors by mosapride consistently enhances the propulsion of the intraluminal content in the distal colon of rats and mice $[10,11]$ while prucalopride, a $5-\mathrm{HT}_{4}$-receptor agonist, induces motility of the entire colon of the dog $[12,13]$ and mediates relaxation and inhibition of spontaneous contractions of the colonic circular muscle with concurrent enhancement of longitudinal muscle contractility in men $[8,14]$. Administration of a non-selective, strong 5- $\mathrm{HT}_{3}$ and weak $5-\mathrm{HT}_{4}$-receptor agonist, Cisapride (Janssen), increases motor activity, while the blockade of $5-\mathrm{HT}_{3}$ and $5-\mathrm{HT}_{4}$ receptors decreases the frequency of migrating motor complexes in the isolated murine terminal ileum and colon [15]. Application of a mixed 5- $\mathrm{HT}_{4}$ receptor agonist and weak 5- $\mathrm{HT}_{3}$-receptors antagonist, TS-951, causes a concentration dependent increase in the amplitude of electrically induced sub-maximal twitch contractions of the longitudinal muscle of the ileum and distal colon of guinea-pig [15].

Acetylcholine is a major neurotransmitter in the enteric nervous system and the gut per se. Its effects are mediated by nicotinic (n) and muscarinic $(\mu)$ receptors. The nACh receptors are non-selective ionotropic receptors, whereas $\mu \mathrm{ACh}$ receptors are part of metabotropic-Gprotein coupled receptors family. Activation of nACh receptors results in the generation of an inward calcium current with the production of fast (fEPSP) or slow (sEPSP) excitatory postsynaptic potentials. In contrast to the diverse effects of serotonin in the gastrointestinal tract, ACh always has an excitatory effect and enhances myoelectrical activity.

\section{Model assumptions}

The general topology of the intercellular arrangements is incorporated in a spatial-temporal model of a segment of the gut-functional unit. The model accurately replicates anatomical, histomorphological and neurobiological data about its peristaltic and propulsive activity. This work is part of a major project on mathematical modeling and computer simulation of gastrointestinal motility and propulsion of a solid non-deformable bolus, in particular. General physiological facts, mathematical formulation of the model, derivation of basic equations, and construction and validation of the numerical algorithm, have been deliberately omitted to avoid unnecessary repetition since these have been extensively discussed in our previous publications [16-21]. The reader is advised to consult the above papers for full details. A brief outline of the general features of the model and numerical algorithm are only given.

Consider the functional unit that contains a solid non-deformable bolus (figure 1). The biomechanical principles of organization of a functional unit are specified as the following modeling assumptions:

i) the FU is modeled as a thin soft orthotropic biological shell;

ii) the wall of the bioshell is composed of two muscle layers, embedded into the connective tissue network; both muscle layers are electrogenic two-dimensional bisyncytia with electrical cable properties; the outer, longitudinal, layer exhibits anisotropic and the inner, circular, layer has isotropic electrical properties; electromechanical coupling in muscle layers with the generation of deformation and forces are considered as a consequence of the evolution of the excitatory wave; 


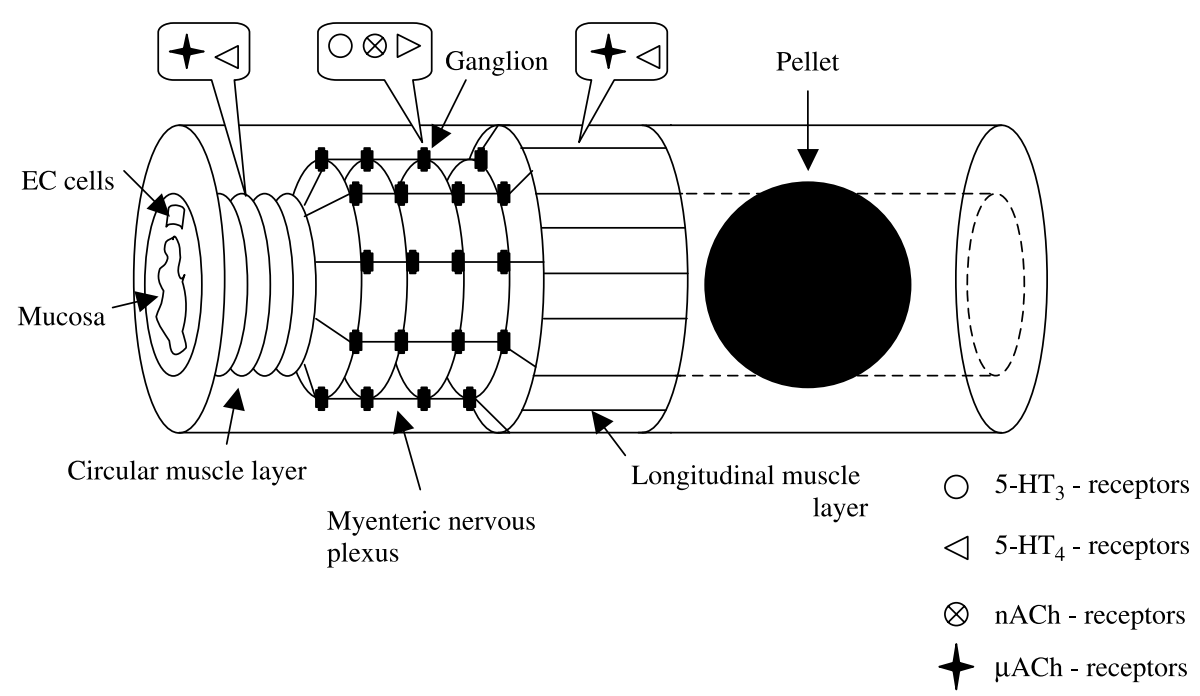

Figure 1. Schematic diagrams of the spatio-temporal model used in numerical experiments. The distribution of serotonergic and cholinergic receptors is as indicated.

iii) the electrical activity of the myogenic syncytium, either slow wave or bursting, represents the integrated function of ion channels: $\mathrm{Ca}^{2+}$ channels of $L$ - and $T$-types, $\mathrm{Ca}^{2+}-K^{+}, K^{+}$, and $\mathrm{Cl}^{-}$channels;

iv) excitation-contraction coupling assumes that the "internal" $\mathrm{Ca}^{2+}$, which enters the cell during the period of excitation, activates the contractile protein system;

v) the mechanical activity and electrical stability of the FU is under the control of the nervous plexus; the latter is modeled as a planar neuronal network; the primary constructive elements of the plexus are: primary sensory (AH-type), motor (S-type) neurons, interneurons (AH/S-type) and a pacemaker cell that is represented by interstitial cells of Cajal (ICC); they discharge electrical potentials of high amplitude at a constant frequency; the electrical behavior of the ICC includes salient morphological and physiological characteristics: the soma, the axon and the nerve terminal; the electrical activity of the soma represents the integrated function of the following ion channels: voltage-dependent $\mathrm{Ca}^{2+}$ channels of $L$ - and $T$ - types, $\mathrm{Ca}^{2+}-\mathrm{K}^{+}, \mathrm{K}^{+}, \mathrm{Na}^{+}$ and leak $\mathrm{Cl}^{-}$channels and mathematically satisfies the Hodgkin-Huxley formalism;

vi) electrochemical synapses are modeled as a null-dimensional three-compartment open pharmacokinetic model (the presynaptic terminal, synaptic cleft and postsynaptic membrane); all chemical reactions of neurotransmitter transformation are adequately described by first order Michaelis-Menten kinetics; synapses contain acetylcholine as a major neurotransmitter and a small amount of co-localized serotonin; the major source of 5-HT is released from enterochromaffin cells though as a result of mechanical stimulation of the wall of the gut;

vii) the dynamics of nerve-pulse transmission and electrochemical coupling in the synapse comprises the following reactions: activation of $\mathrm{Ca}^{2+}$ influx into the presynaptic terminal through voltage-gated channels; neurotransmitter release from the stores; diffusion of the free fraction into the synaptic cleft; binding with receptors located on the postsynaptic membrane; utilization of unreacted neurotransmitter by the enzyme(s); and fast excitatory postsynaptic potential (fEPSP) generation; 
viii) the link between the ICC and smooth muscle syncytia is made possible through the $L$-type voltage-dependent $\mathrm{Ca}^{2+}$ channels on the smooth muscle. The effect is assumed to be mainly chronotropic with an increase in the time of permeability for calcium ions;

ix) motion of a solid non-deformable spherical pellet is due to the propagation of the electromechanical wave of deformation along the segment of the gut and is subjected to dry and viscous friction; we assume that the contact forces between the wall of the bioshell and the pellet are orthogonal to the surface of the pellet.

\section{Mathematical formulation}

The equation of the balance of momentum of a segment (functional unit) of the gut is:

$$
\begin{aligned}
\gamma_{0}\left(\frac{\partial v_{r}}{\partial t}-\frac{v_{s}^{2}}{r}\right)= & \frac{\partial}{\partial \tilde{s}_{1}}\left[\left(\kappa \frac{\partial\left(\lambda_{c}-1\right)}{\partial t}+T^{a}\left(\lambda_{c}\right)+T^{p}\left(\lambda_{c}, \lambda_{l}\right)\right) \mathrm{e}_{1 r} \sqrt{g_{22}}\right] \\
& +\frac{\partial}{\partial \tilde{s}_{2}}\left[\left(\kappa \frac{\partial\left(\lambda_{l}-1\right)}{\partial t}+T^{a}\left(\lambda_{l}\right)+T^{p}\left(\lambda_{c}, \lambda_{l}\right)\right) \mathrm{e}_{2 r} \sqrt{g_{11}}\right]+p \sqrt{g} n_{r}, \\
\gamma_{0}\left(\frac{\partial v_{s}}{\partial t}-\frac{v_{s} v_{r}}{r}\right)= & \frac{\partial}{\partial \tilde{s}_{1}}\left[\left(\kappa \frac{\partial\left(\lambda_{c}-1\right)}{\partial t}+T^{a}\left(\lambda_{c}\right)+T^{p}\left(\lambda_{c}, \lambda_{l}\right)\right) \mathrm{e}_{1 s} \sqrt{g_{22}}\right] \\
& +\frac{\partial}{\partial \tilde{s}_{2}}\left[\left(\kappa \frac{\partial\left(\lambda_{l}-1\right)}{\partial t}+T^{a}\left(\lambda_{l}\right)+T^{p}\left(\lambda_{c}, \lambda_{l}\right)\right) \mathrm{e}_{2 s} \sqrt{g_{11}}\right]+p \sqrt{g} n_{s}, \\
\gamma_{0} \frac{\partial v_{z}}{\partial t}= & \frac{\partial}{\partial \tilde{s}_{1}}\left[\left(\kappa \frac{\partial\left(\lambda_{c}-1\right)}{\partial t}+T^{a}\left(\lambda_{c}\right)+T^{p}\left(\lambda_{c}, \lambda_{l}\right)\right) \mathrm{e}_{1 z} \sqrt{g_{22}}\right] \\
& +\frac{\partial}{\partial \tilde{s}_{2}}\left[\left(\kappa \frac{\partial\left(\lambda_{l}-1\right)}{\partial t}+T^{a}\left(\lambda_{l}\right)+T^{p}\left(\lambda_{c}, \lambda_{l}\right)\right) \mathrm{e}_{2 z} \sqrt{g_{11}}\right]+p \sqrt{g} n_{z}, \\
& \left(0 \leq \tilde{s}_{1} \leq 2 \pi r, 0 \leq \tilde{s}_{2} \leq l\right)
\end{aligned}
$$

where the radial $\left(v_{r}\right)$, circumferential $\left(v_{s}\right)$ and longitudinal components $\left(v_{z}\right)$ of the wall velocity vector are:

$$
v_{r}=\frac{\mathrm{d} r}{\mathrm{~d} t}, \quad v_{s}=\frac{\mathrm{d} s}{\mathrm{~d} t}, \quad v_{z}=\frac{\mathrm{d} z}{\mathrm{~d} t},
$$

the rate of elongation (hereafter, the subscripts $(l)$ and $(c)$ are related to the longitudinal and circular muscle layers, respectively) is:

$$
\lambda_{c}=\frac{\mathrm{d} s}{\mathrm{~d} \tilde{s}_{1}}, \quad \lambda_{l}=\frac{\mathrm{d} z}{\mathrm{~d} \tilde{s}_{2}}
$$

The components and determinant of the fundamental metric tensor are:

$$
g_{i j}=\frac{\partial r}{\partial \tilde{s}_{i}} \frac{\partial r}{\partial \tilde{s}_{j}}+\frac{\partial s}{\partial \tilde{s}_{i}} \frac{\partial s}{\partial \tilde{s}_{j}}+\frac{\partial z}{\partial \tilde{s}_{i}} \frac{\partial z}{\partial \tilde{s}_{j}} \quad g=g_{11} g_{22}-g_{12}^{2}
$$


and the direction cosines of the outward normal $n$ vector to the surface with respect to the cylindrical $j$-axis $(i=1,2 ; j=r, s, z)$ are calculated as:

$$
\begin{aligned}
& \mathrm{e}_{i r}=\frac{1}{\sqrt{g_{i i}}} \frac{\partial r}{\partial \tilde{s}_{i}}, \quad \mathrm{e}_{i s}=\frac{1}{\sqrt{g_{i i}}} \frac{\partial s}{\partial \tilde{s}_{i}}, \quad \mathrm{e}_{i z}=\frac{1}{\sqrt{g_{i i}}} \frac{\partial z}{\partial \tilde{s}_{i}}, \\
& n_{r}=\left(\mathrm{e}_{1 s} \mathrm{e}_{2 s}-\mathrm{e}_{1 z} \mathrm{e}_{2 z}\right) \sqrt{g_{11}} \sqrt{g_{22}} / \sqrt{g}, \\
& n_{s}=\left(\mathrm{e}_{1 z} \mathrm{e}_{2 r}-\mathrm{e}_{1 r} \mathrm{e}_{2 s}\right) \sqrt{g_{11}} \sqrt{g_{22}} / \sqrt{g}, \\
& n_{r}=\left(\mathrm{e}_{1 r} \mathrm{e}_{2 s}-\mathrm{e}_{1 s} \mathrm{e}_{2 r}\right) \sqrt{g_{11}} \sqrt{g_{22}} / \sqrt{g} .
\end{aligned}
$$

Here, the following notations are used: $\gamma_{0}$ is the linear density of a biomaterial in an undeformed state, $T^{p}, T^{a}$ are the passive and active components, respectively, of the total force $\left(T_{c, l}^{p}\right)$ of the wall, $\kappa$ is a rheological parameter, $p$ is intraluminal pressure, $\varphi_{l}, \varphi_{c}$ are the electrical waves of depolarization of the longitudinal and circular muscle layers, and $\tilde{s}_{1}, \tilde{s}_{2}$ are Lagrangian coordinates of the bioshell.

The passive $\left(T_{c, l}^{p}\right)$ components are calculated from:

$$
T_{\mathrm{Cl}}^{p}=\frac{\partial \gamma_{0} W}{\partial\left(\lambda_{\mathrm{Cl}}-1\right)}
$$

where $W$ is the strain energy density function of the connective tissue and passive muscular components:

$$
\begin{aligned}
\gamma_{0} W= & \frac{1}{2}\left[c_{1}\left(\lambda_{l}-1\right)^{2}+2 c_{3}\left(\lambda_{l}-1\right)\left(\lambda_{c}-1\right)+c_{2}\left(\lambda_{c}-1\right)^{2}\right. \\
& \left.+c_{7} \exp \left(c_{4}\left(\lambda_{l}-1\right)^{2}+c_{5}\left(\lambda_{c}-1\right)^{2}+2 c_{6}\left(\lambda_{l}-1\right)\left(\lambda_{c}-1\right)\right)\right] .
\end{aligned}
$$

For the active force $\left(T_{c, l}^{a}\right)$ components, we assume the empirical relationship:

$$
T_{c, l}^{a}= \begin{cases}0, & {\left[\mathrm{Ca}^{2+}\right] \leq 0.1 \mu \mathrm{M}} \\ c_{8(c, l)}+c_{9(c, l)}\left[\mathrm{Ca}^{2+}\right]^{4}+c_{10(c, l)}\left[\mathrm{Ca}^{2+}\right]^{3} & \\ +c_{11(c, l)}\left[\mathrm{Ca}^{2+}\right]^{2}+c_{12(c, l)}\left[\mathrm{Ca}^{2+}\right], & 0.1<\left[\mathrm{Ca}^{2+}\right] \leq 1 \mu \mathrm{M} \\ \max T^{a}, & {\left[\mathrm{Ca}^{2+}\right]>1 \mu \mathrm{M}}\end{cases}
$$

where $T_{c, l}^{a}$ is the active force generated by the smooth muscle syncytium, and $c_{1-12(c, l)}$ are the mechanical constants.

The characteristic feature of soft shells is the possibility for the simultaneous coexistence of unstressed, uniaxial and biaxial stress-strained zones. The creaseless shape occurs at $\lambda_{l, c}>1.0$; in the case of the development of creases, when $\lambda_{l} \leq 1.0$ or $\lambda_{c} \leq 1.0$, the wrinkled area is modeled by a "smooth zone" made up by the system of unbound filaments. This is determined by requirements that are geometrically related to the conservation of smoothness of the surface of the bioshell, and force conditions related to the continuity of membrane forces. Thus, if creases appear on the surface of the bioshell, then for $T_{c, l}^{p}$ at the 
uniaxial stress-strained state we assume:

$$
T_{(c, l)}^{p}= \begin{cases}0, & \lambda_{(c, l)} \leq 1, \\ c_{13(c, l)}\left\{\exp \left[c_{14(c, l)}\left(\lambda_{(c, l)}-1\right)\right]-1\right\}, & \lambda_{(c, l)}>1 .\end{cases}
$$

Here: $c_{13,14(c, l)}$ are the empirical mechanical constants of the wall of the gut.

The dynamics of propagation of the electrical wave $\varphi_{l}$ along the anisotropic longitudinal muscle layer is defined as:

$$
C_{m} \frac{\partial \varphi_{l}}{\partial t}=I_{m 1}\left(\tilde{s}_{1}, \tilde{s}_{2}\right)+I_{m 2}\left(\tilde{s}_{1}, \tilde{s}_{2}\right)+I_{\text {ionic }}^{*}
$$

where $I_{m 1}, I_{m 2}$ are the transmembrane currents per unit volume:

$$
\begin{aligned}
& I_{m 1}\left(\tilde{s}_{1}, \tilde{s}_{2}\right)=M_{v s}\left\{-\frac{2\left(\mu_{s_{2}}-\mu_{s_{1}}\right)}{\left(1+\mu_{s_{1}}\right)\left(1+\mu_{s_{2}}\right)} \arctan \left(\frac{\mathrm{d} \tilde{s}_{1}}{\mathrm{~d} \tilde{s}_{2}} \sqrt{\frac{G_{s_{2}}}{G_{s_{1}}}}\right)+\frac{g_{0, s_{1}}}{G_{s_{1}}}\right\} \\
& \times\left(\frac{\partial}{\partial \tilde{s}_{1}}\left(\frac{g_{0, s_{1}}}{\lambda_{c}} \frac{\partial \varphi_{l}}{\partial \tilde{s}_{1}}\right)+\frac{\partial}{\partial \tilde{s}_{2}}\left(\frac{g_{0, s_{2}}^{\cdot}}{\lambda_{l}} \frac{\partial \varphi_{l}}{\partial \tilde{s}_{2}}\right)\right), \\
& I_{m 2}\left(\tilde{s}_{1}, \tilde{s}_{2}\right)=M_{v s} \iint_{S} \frac{\left(\mu_{\tilde{s}_{1}}-\mu_{\tilde{s}_{2}}\right)}{2 \pi\left(1+\mu_{\tilde{s}_{1}}\right)\left(1+\mu_{\tilde{s}_{2}}\right)} \frac{\left(\tilde{s}_{2}-\tilde{s}_{2}^{\prime}\right) / G_{\tilde{s}_{2}}-\left(\tilde{s}_{1}-\tilde{s}_{1}^{\prime}\right) / G_{\tilde{s}_{1}}}{\left[\left(\tilde{s}_{1}-\tilde{s}_{1}^{\prime}\right) / G_{\tilde{s}_{1}}-\left(\tilde{s}_{2}-\tilde{s}_{2}^{\prime}\right) / G_{\tilde{s}_{2}}\right]^{2}} \\
& \times\left[\frac{\partial}{\partial \tilde{s}_{1}}\left(\frac{g_{0, \tilde{s}_{1}}^{*}}{\lambda_{c}} \frac{\partial \varphi_{l}}{\partial \tilde{s}_{1}}\right)+\frac{\partial}{\partial \tilde{s}_{2}}\left(\frac{g_{0, \tilde{s}_{2}}^{*}}{\lambda_{l}} \frac{\partial \varphi_{l}}{\partial \tilde{s}_{2}}\right)\right] \mathrm{d} \tilde{s}_{1}^{\prime} \mathrm{d} \tilde{s}_{2}^{\prime}, \\
& \mu_{\tilde{s}_{1}}=g_{0, \tilde{s}_{1}}^{*} / g_{i, \tilde{s}_{1}}^{*}, \quad \mu_{\tilde{s}_{2}}=g_{0, \tilde{s}_{2}}^{*} / g_{i, \tilde{s}_{2}}^{*}, \\
& G_{\tilde{s}_{1}}=\frac{g_{0, \tilde{s}_{1}}^{*}+g_{i, \tilde{s}_{1}}^{*}}{\lambda_{c}}, G_{\tilde{s}_{2}}=\frac{g_{0, \tilde{s}_{2}}^{*}+g_{i, \tilde{s}_{2}}^{*}}{\lambda_{l}}, G=\sqrt{G_{\tilde{s}_{1}} G_{\tilde{s}_{2}}},
\end{aligned}
$$

and the following notations are used: $C_{m}$ is the capacitance of smooth muscle, $g_{i, \tilde{s}_{1}}^{*}, g_{i, \tilde{s}_{1}}^{*}, g_{0, \tilde{s}_{1}}^{*}$, $g_{0, \tilde{s}_{2}}^{*}$ are the maximal intracellular (the subscript $(i)$ ) and interstitial space (the subscript (0)) conductivities of the longitudinal and circular muscle layers in the longitudinal and circumferential directions, respectively, $M_{v s}$ is the membrane volume-to-surface ratio, and $I_{\text {ionic }}^{*}$ is the total ionic current:

$$
I_{\text {ionic }}^{*}=\bar{g}_{\mathrm{Na}} m^{* 3} h^{*}\left(\varphi_{(c, l)}-\bar{\varphi}_{\mathrm{Na}}\right)+\bar{g}_{\mathrm{K}} n^{* 4}\left(\varphi_{(c, l)}-\bar{\varphi}_{\mathrm{K}}\right)+\bar{g}_{\theta}\left(\varphi_{(c, l)}-\bar{\varphi}_{\theta}\right) .
$$

$\bar{g}_{\mathrm{Na}}, \bar{g}_{\mathrm{K}}, \bar{g}_{\theta}$ are the maximal conductances, $m^{*}, h^{*}$, and $n^{*}$ are the probabilities of opening of the ion gates, and $\bar{\varphi}_{\mathrm{Na}}, \bar{\varphi}_{\mathrm{K}}, \bar{\varphi}_{\theta}$ are the reversal potentials of $\mathrm{Na}^{+}, \mathrm{K}^{+}$, and $\mathrm{Cl}^{-}$currents, respectively, in smooth muscles.

The dynamics of change in the probability variables can be obtained from the solution of the first-order equation:

$$
\frac{\mathrm{d} y}{\mathrm{~d} t}=\alpha_{y}^{*}(1-y)-\beta_{y}^{*} y,
$$


where $y$ refers to $m^{*}, h^{*}$ and $n^{*}$, respectively. The activation, $\alpha_{y}^{*}$, and deactivation, $\beta_{y}^{*}$, parameters for smooth muscle satisfy the following relations:

$$
\begin{aligned}
\alpha_{m}^{*} & =0.005\left(\varphi_{(c, l)}-\bar{\varphi}_{m}\right) /\left\{\exp \left[0.1\left(\varphi_{(c, l)}-\bar{\varphi}_{m}\right)\right]-1\right\}, \\
\alpha_{h}^{*} & =0.014 \exp \left[-\left(\bar{\varphi}_{h}+\varphi_{(c, l)}\right) / 20\right], \\
\alpha_{n}^{*} & =0.006\left(\varphi_{(c, l)}-\bar{\varphi}_{n}\right) /\left\{\exp \left[0.1\left(\varphi_{(c, l)}-\bar{\varphi}_{n}\right)\right]-1\right\}, \\
\beta_{m}^{*} & =0.2 \exp \left[\left(\varphi_{(c, l)}+\bar{\varphi}_{m}\right) / 38\right], \\
\beta_{h}^{*} & =0.2 /\left\{1+\exp \left[0.2\left(\bar{\varphi}_{h}-\varphi_{(c, l)}\right)\right]\right\}, \\
\beta_{n}^{*} & =0.75 \exp \left(\bar{\varphi}_{n}-\varphi_{(c, l)}\right),
\end{aligned}
$$

The dynamics of propagation of the electrical wave $\varphi_{c}$ along the isotropic circular muscle layer are:

$$
C_{m} \frac{\partial \varphi_{c}}{\partial t}=\frac{M_{v s}}{1+\mu_{\tilde{s}_{1}}}\left[\frac{\partial}{\partial \tilde{s}_{1}}\left(\frac{g_{0, \tilde{s}_{1}}^{*}}{\lambda_{c}} \frac{\partial \varphi_{c}}{\partial \tilde{s}_{1}}\right)+\frac{\partial}{\partial \tilde{s}_{2}}\left(\frac{g_{0, \tilde{s}_{1}}^{*}}{\lambda_{l}} \frac{\partial \varphi_{c}}{\partial \tilde{s}_{2}}\right)\right]-I_{\text {ionic }}^{*}
$$

where the above-mentioned abbreviations and relationships for $I_{\text {ionic }}^{*}$ are used.

The intraluminal pressure $p$ is assumed to be uniform and changes according to the adiabatic law:

$$
p=p_{0} \Delta V^{1.41},
$$

$p_{0}$ is the value of $p$ at $t=0 ; \Delta V$ is the ratio of the present $(V)$ intraluminal volume of the bioshell to its initial value $\left(V_{0}\right)$, i.e. $\Delta V=V_{0} / V$.

The myoelectrical activity in a locus of the small bowel is governed by the dynamics of voltage-dependent $\mathrm{Ca}^{2+}$ channels ( $L$ - and $T$-type), mixed $\mathrm{Ca}^{2+}-\mathrm{K}^{+}$and $\mathrm{K}^{+}$channels and chloride channels. Thus, the system of equations is a linear sum of the five ionic currents:

$$
\alpha C_{m} \frac{\mathrm{d} \varphi}{\mathrm{d} t}=-\left(I_{\mathrm{Ca}}^{f}+I_{\mathrm{Ca}}^{s}+I_{\mathrm{Ca}-\mathrm{K}}^{*}+I_{\mathrm{K}}^{*}+I_{\mathrm{Cl}}^{*}\right) .
$$

Here, $C_{m}$ is the membrane capacitance, $\varphi$ is the membrane potential and $I_{(i)}$ are ionic currents carried by different ions and defined as $I_{\mathrm{Ca}}^{f}, I_{\mathrm{Ca}}^{s}$-the fast and slow inward calcium currents via $T$ - and $L$-type voltage-dependent $\mathrm{Ca}^{2+}$ channels, respectively:

$$
I_{\mathrm{Ca}}^{f}=\tilde{g}_{\mathrm{Ca}}^{f} \tilde{m}_{I}^{3} \tilde{h}\left(\varphi-\tilde{\varphi}_{\mathrm{Ca}}^{*}\right), \quad I_{\mathrm{Ca}}^{s}=\tilde{g}_{\mathrm{Ca}}^{s} \tilde{x}_{\mathrm{Ca}}\left(\varphi-\tilde{\varphi}_{\mathrm{Ca}}^{*}\right) .
$$

$I_{\mathrm{Ca}-\mathrm{K}}^{*}, I_{\mathrm{Cl}}^{*}$ are the outward calcium activated potassium and voltage-activated potassium currents, respectively:

$$
I_{\mathrm{K}}^{*}=\tilde{g}_{\mathrm{K}}^{*} \tilde{n}^{4}\left(\varphi-\tilde{\varphi}_{\mathrm{K}}^{*}\right), \quad I_{\mathrm{Ca}-\mathrm{K}}^{*}=\tilde{g}_{\mathrm{Ca}-\mathrm{K}}^{*}\left[\mathrm{Ca}^{2+}\right]\left(\varphi-\tilde{\varphi}_{\mathrm{K}}^{*}\right) /\left(0.5+\left[\mathrm{Ca}^{2+}\right]\right)
$$

and $I_{\mathrm{Cl}}^{*}$ is the leak chloride current:

$$
I_{\mathrm{Cl}}^{*}=\tilde{g}_{\mathrm{Cl}}^{*}\left(\varphi-\tilde{\varphi}_{\mathrm{Cl}}^{*}\right),
$$

where: $\tilde{\varphi}_{\mathrm{Ca}}^{*}, \tilde{\varphi}_{\mathrm{K}}^{*}, \tilde{\varphi}_{\mathrm{Cl}}^{*}$ are the reversal potentials for the respective currents, $\left[\mathrm{Ca}^{2+}\right]$ is the intracellular concentration of calcium ions in the functional unit, $\tilde{g}_{\mathrm{Ca}}^{f}, \tilde{g}_{\mathrm{Ca}}^{s}, \tilde{g}_{\mathrm{K}}^{*}, \tilde{g}_{\mathrm{Ca}-\mathrm{K}}^{*}, \tilde{g}_{\mathrm{Cl}}^{*}$ are the maximal conductances of voltage-dependent $\mathrm{Ca}^{2+}, \mathrm{Ca}^{2+}$-activated $\mathrm{K}^{+}$channels and 
leak $\mathrm{Cl}^{-}$conductance, $\tilde{m}, \tilde{h}, \tilde{n}$ and $\tilde{x}_{\mathrm{Ca}}$ are dynamic variables of these channels whose kinetics is described by:

$$
\begin{aligned}
\tilde{m}_{I} & =\tilde{\alpha}_{m} /\left(\tilde{\alpha}_{m}+\tilde{\beta}_{m}\right), \\
\frac{\mathrm{d} \tilde{h}}{\mathrm{~d} t} & =\left[\tilde{\alpha}_{h}(1-\tilde{h})-\tilde{\beta}_{h} \tilde{h}\right] / \alpha \lambda, \\
\frac{\mathrm{d} \tilde{n}}{\mathrm{~d} t} & =\left[\tilde{\alpha}_{n}(1-\tilde{n})-\tilde{\beta}_{n} \tilde{n}\right] / \alpha \lambda, \\
\frac{\mathrm{d} \tilde{x}_{\mathrm{Ca}}}{\mathrm{d} t} & =\left\{1+[\exp [-0.15(\varphi+50)]]^{-1}-\tilde{x}_{\mathrm{Ca}}\right\} / \alpha \tau_{\tilde{x}_{\mathrm{Ca}}}, \\
\frac{\mathrm{dCa} a^{2+}}{\mathrm{d} t} & =\frac{\rho}{\alpha}\left[\mathrm{K}_{c} \tilde{x}_{\mathrm{Ca}}\left(\bar{\varphi}_{\mathrm{Ca}}-\varphi\right)-\left[\mathrm{Ca}^{2+}\right]\right],
\end{aligned}
$$

where

$$
\begin{aligned}
\tilde{\alpha}_{m} & =0.1(50-\tilde{\varphi}) /[\exp (5-0.1 \tilde{\varphi})-1], \\
\tilde{\alpha}_{h} & =0.07 \exp [(25-0.1 \tilde{\varphi}) / 20], \\
\tilde{\alpha}_{n} & =0.01(55-\tilde{\varphi}) /\{\exp [(55-\tilde{\varphi}) / 10]-1\}, \\
\tilde{\beta}_{m} & =4 \exp [(25-\tilde{\varphi}) / 18], \\
\tilde{\beta}_{h} & =1 /[1+\exp (5.5-0.1 \tilde{\varphi})], \\
\tilde{\beta}_{n} & =0.125 \exp [(45-\tilde{\varphi}) / 80] .
\end{aligned}
$$

Here, $\tilde{\varphi}=(127 \varphi+8265) / 105, \tau_{x_{\mathrm{Ca}}}$ is the time constant and $\rho, \mathrm{K}_{c}$ are parameters referring to the dynamics of calcium channels, $\alpha$ and $\lambda$ are numerical constants, and parameters $\tilde{\alpha}$ and $\tilde{\beta}$ have the same meaning as above.

The evolution of $L$-type voltage-dependent $\mathrm{Ca}^{2+}$ - channels is

$$
\tilde{g}_{\mathrm{Ca}}^{s}=\delta\left(\varphi_{p}^{+}\right) \tilde{g}_{\mathrm{Ca}}^{s},
$$

where $\delta\left(\varphi_{p}^{+}\right)$is the function defined as $\delta\left(\varphi_{p}^{+}\right)=1$ when $\delta\left(\varphi_{p}^{+}\right) \geq \varphi_{p}^{*}$-threshold value, and $\delta\left(\varphi_{p}^{+}\right)=0$ otherwise.

Dynamics of the discharge of interstitial cell of Cajal $\varphi_{(i)}$ is described by:

$$
C_{s} \mathrm{~d} \varphi_{(i)} / \mathrm{d} t=-\left(I_{\mathrm{Ca}}+I_{\mathrm{Ca}-\mathrm{K}}+I_{\mathrm{Na}}+I_{\mathrm{K}}+I_{\mathrm{Cl}}\right)+I_{\mathrm{ext}(i)}
$$

where: $C_{S}$ is the membrane capacitance, $I_{\ldots}$ are ionic currents carried through different ionic channels and $I_{\mathrm{ext}(i)}$ is the external membrane current. The equations for ionic currents are:

$$
\begin{aligned}
& I_{\mathrm{Ca}}=g_{\mathrm{Ca}(i)} z /\left(1+\zeta\left[\mathrm{Ca}^{2+}\right]_{n}\right)\left(\varphi_{(i)}-\varphi_{\mathrm{Ca}}\right) \\
& I_{\mathrm{Ca}-\mathrm{K}}=g_{\mathrm{Ca}-\mathrm{K}} \rho_{\infty}\left[\mathrm{Ca}^{2+}\right]_{n} /\left(0.5+\left[\mathrm{Ca}^{2+}\right]_{n}\right)\left(\varphi_{(i)}-\varphi_{\mathrm{Ca}-\mathrm{K}}\right) \\
& +g_{\mathrm{Na}(i)} m_{\mathrm{Na}}^{3} h_{\mathrm{Na}}\left(\varphi_{(i)}-\varphi_{\mathrm{Na}}\right) \\
& I_{\mathrm{K}}=g_{\mathrm{K}} n_{\mathrm{K}}^{4}\left(\varphi_{(i)}-\varphi_{\mathrm{K}}\right) \\
& I_{\mathrm{Cl}}=g_{\mathrm{Cl}(i)}\left(\varphi_{(i)}-\varphi_{\mathrm{Cl}}\right) \text {, }
\end{aligned}
$$

here: $\varphi_{\mathrm{Ca}}, \varphi_{\mathrm{Ca}-\mathrm{K}}, \varphi_{\mathrm{Na}}, \varphi_{\mathrm{K}}, \varphi_{\mathrm{Cl}}$ are the reversal potentials for the respective currents, $g_{\mathrm{Ca}(i)}$, $g_{\mathrm{Ca}-\mathrm{K}}, g_{\mathrm{Na}(i)}, g_{\mathrm{K}}, g_{\mathrm{Cl}(i)}$ are the maximal conductances of voltage-dependent $\mathrm{Ca}^{2+}$ - channels ( $N$-type), $\mathrm{Ca}^{2+}$-activated $\mathrm{K}^{+}$channels, $\mathrm{Na}^{+}, \mathrm{K}^{+}$and leak $\mathrm{Cl}^{-}$channels, $\xi$ is the specific 
parameter of calcium inhibition of the $\mathrm{Ca}^{2+}$ channels, $\left[\mathrm{Ca}^{2+}\right]_{n}$ is the intracellular concentration of free calcium, and $z, \rho_{\infty}, m_{\mathrm{Na}}, h_{\mathrm{Na}}$ and $n_{\mathrm{K}}$ are dynamic variables of the ionic channels whose kinetics is described as:

$$
\begin{aligned}
\mathrm{d} z / \mathrm{d} t & =\left(z_{\infty}-z\right) / \tau_{z} \\
\mathrm{~d} h_{\mathrm{Na}} / \mathrm{d} t & =\lambda_{h}\left(h_{\infty}-h_{\mathrm{Na}}\right) / \tau_{h} \\
\mathrm{~d} n_{\mathrm{K}} / \mathrm{d} t & =\lambda_{n}\left(n_{\infty}-n_{\mathrm{K}}\right) / \tau_{h} \\
\rho_{\infty} & =1 /\left\{1+\exp \left[0.15\left(\varphi_{(i)}+47\right)\right]\right\} \\
m_{\mathrm{Na}} & =m_{\infty}\left(\varphi_{(i)}\right) \\
y_{\infty} & =\alpha_{y \infty} \tau_{y}\left(y=m_{\mathrm{Na}}, h_{\mathrm{Na}}, n_{\mathrm{K}}\right) \\
\tau_{y} & =1 /\left(\alpha_{y \infty}+\beta_{y \infty}\right) \\
\alpha_{m \infty} & =0.12\left(\varphi_{(i)}+27\right) /\left\{1-\exp \left[-\left(\varphi_{(i)}+27\right) / 8\right]\right\} \\
\beta_{m \infty} & =4 \exp \left[-\left(\varphi_{(i)}+47\right) / 15\right] \\
\alpha_{h \infty} & =0.07 \exp \left[-\left(\varphi_{(i)}+47\right) / 17\right] \\
\beta_{h \infty} & =1 /\left\{1+\exp \left[-\left(\varphi_{(i)}+22\right) / 8\right]\right\} \\
\alpha_{n \infty} & =0.012\left(\varphi_{(i)}+12\right) /\left\{1-\exp \left[-\left(\varphi_{(i)}+12\right) / 8\right]\right\} \\
\beta_{n \infty} & =0.125 \exp \left[-\left(\varphi_{(i)}+20\right) / 67\right] \\
z_{\infty} & =1 /\left\{1+\exp \left[-0.15\left(\varphi_{(i)}+42\right)\right]\right\} .
\end{aligned}
$$

Changes in the free cytosolic calcium concentration yield:

$$
\mathrm{d}\left[\mathrm{Ca}^{2+}\right]_{n} / \mathrm{d} t=0.2 z\left(\varphi_{\mathrm{Ca}}-\varphi_{(i)}\right) /\left(1+\zeta\left[\mathrm{Ca}^{2+}\right]_{n}\right)-0.3\left[\mathrm{Ca}^{2+}\right]_{n} .
$$

The membrane current is $I_{\mathrm{ext}(i)}=\varphi_{(i)} / R$ where $R$ is the input resistance.

The system of kinetic equations of the cycle of chemical transformations of acetylcholine at the cholinergic synapses is as follows:

$$
\begin{aligned}
\mathrm{d}\left[\mathrm{Ca}^{2+}\right]_{c} / \mathrm{d} t= & {\left[\mathrm{Ca}^{2+}\right]_{\mathrm{out}} \varphi_{(i)}-k_{+5}\left[\mathrm{Ca}^{2+}\right]_{c} } \\
\mathrm{~d}\left[\mathrm{ACh}_{v}\right] / \mathrm{d} t= & -k_{c}\left[\mathrm{Ca}^{2+}\right]_{c}\left[\mathrm{ACh}_{v}\right] \\
\mathrm{d}\left[\mathrm{ACh}_{f}\right] / \mathrm{d} t= & k_{c}\left[\mathrm{Ca}^{2+}\right]_{c}\left[\mathrm{ACh}_{v}\right]-k_{d}\left[\mathrm{ACh}_{f}\right]+k_{+6}[S] \\
\mathrm{d}\left[\mathrm{ACh}_{c}\right] / \mathrm{d} t= & k_{d}\left[\mathrm{ACh}_{f}\right]-k_{+p}\left[\mathrm{ACh}_{c}\right] \\
\mathrm{d}\left[\mathrm{ACh}_{p}\right] / \mathrm{d} t= & k_{+p}\left[\mathrm{ACh}_{c}\right]-\left[\mathrm{ACh}_{p}\right]\left(k_{+1}\left[R^{0}\right]+k_{+2}\left[E^{0}\right]\right) \\
& +\left[\mathrm{ACh}_{p}\right]\left(k_{+1}[\mathrm{ACh}-R]+k_{+2}[\mathrm{AChE}]\right) \\
& +k_{-1}[\mathrm{ACh}-R]+k_{-2}[\mathrm{AChE}] \\
\mathrm{d}[\mathrm{ACh}-R] / \mathrm{d} t= & k_{+1}\left[\mathrm{ACh}_{p}\right]\left[R^{0}\right]-[\mathrm{ACh}-R]\left(k_{-1}+k_{+4}+k_{+1}\left[\mathrm{ACh}_{p}\right]\right) \\
\mathrm{d}[\mathrm{AChE}] / \mathrm{d} t= & k_{+2}\left[E^{0}\right]\left[\mathrm{ACh} h_{p}\right]-[\mathrm{AChE}]\left(k_{-2}+k_{+3}+k_{+2}\left[\mathrm{ACh}_{p}\right]\right) \\
\mathrm{d}[S] / \mathrm{d} t= & k_{+4}[\mathrm{ACh}-R]-k_{+6}[S]+k_{+3}[\mathrm{AChE}] \\
{[R]=} & {\left[R^{0}\right]-[\mathrm{ACh}-R] } \\
{[E]=} & {\left[E^{0}\right]-[\mathrm{AChE}], }
\end{aligned}
$$

where: $k_{-,+}$are the constants of backward and forward chemical reactions, $k_{c}$ is the affinity constant, $k_{d}$ is the constant of diffusion, $k_{+p}$ is the diffusion velocity of $\mathrm{ACh}$ on the 
subsynaptic membrane, $\mathrm{ACh}_{v}$ is vesicular acetylcholine and its free $\left(\mathrm{ACh}_{f}\right)$ and postsynaptic $\left(\mathrm{ACh}_{p}\right)$ fractions, $R$ are receptors, $E$ is acetylcholine-esterase enzyme (superscript 0 indicates the initial concentration), $\mathrm{ACh}-\mathrm{R}$ is acetylcholine-receptor complex, $\mathrm{AChE}$ is acetylcholineenzyme complex, $S$ are products of chemical reactions, and $\left(\mathrm{Ca}^{2+}\right)_{\text {out }}$ is the external calcium ion content.

The dynamics of serotonin (SR) conversion and the activation of serotonin type 4 receptors is:

$$
\begin{aligned}
& \mathrm{d}\left[\mathrm{SR}_{\mathrm{v}}\right] / \mathrm{d} t=-1 / 2 k_{v}\left[\mathrm{Ca}^{2+}\right]_{\mathrm{out}} \varepsilon(t)\left[\mathrm{SR}_{v}\right] \\
& \mathrm{d}\left[\mathrm{SR}_{f}\right] / \mathrm{d} t=k_{v}\left[\mathrm{Ca}^{2+}\right] \varepsilon(t)\left[\mathrm{SR}_{\mathrm{v}}\right]-k_{+2}^{*}\left[\mathrm{SR}_{\mathrm{f}}\right] \\
& \mathrm{d}\left[\mathrm{SR}_{c}\right] / \mathrm{d} t=k_{+2}^{*}\left[\mathrm{SR}_{\mathrm{f}}\right]+\left[\mathrm{SR}_{c}-\mathrm{MAO}\right]\left(k_{-3}^{*}+k_{-3}^{*}\left[\mathrm{SR}_{c}\right]\right) \\
& \left.-\left[\mathrm{SR}_{c}\right]\left(k_{+3}^{*}\left[\mathrm{E}^{0}\right]+k_{+5}^{*}\left[\mathrm{R}_{5-\mathrm{HT} 4}\right]\right)+k_{-5}^{*}\right)\left[\mathrm{SR}_{c}-\mathrm{R}_{5-\mathrm{HT} 4}\right] \\
& \mathrm{d}\left[\mathrm{SR}_{c}-\mathrm{MAO}\right] / \mathrm{d} t=k_{+3}^{*}\left[\mathrm{SR}_{c}\right]\left[\mathrm{MAO}^{0}\right]-\left[\mathrm{SR}_{c}-\mathrm{MAO}\right]\left(k_{-3}^{*}+k_{+4}^{*}+k_{+3}^{*}\left[\mathrm{SR}_{c}\right]\right) \\
& \mathrm{d}\left[\mathrm{SR}_{c}-\mathrm{R}_{5-\mathrm{HT} 4}\right] / \mathrm{d} t=k_{+5}^{*}\left[\mathrm{SR}_{c}\right]\left[\mathrm{R}_{5-\mathrm{HT} 4}\right]-\left[\mathrm{SR}_{c}-\mathrm{R}_{5-\mathrm{HT} 4}\right]\left(k_{-5}^{*}+k_{+6}^{*}+k_{+7}^{*}\left(\left[\mathrm{G}^{0}\right]\right.\right. \\
& \left.-\left[\mathrm{SR}_{c}-\mathrm{R}_{5-\mathrm{HT} 4}-\mathrm{G}\right]\right) \\
& \mathrm{d}\left[\mathrm{R}_{5-\mathrm{HT} 4}\right] / \mathrm{d} t=\left(k_{-5}^{*}+k_{+6}^{*}\right)\left[\mathrm{SR}_{c}-\mathrm{R}_{5-\mathrm{HT} 4}\right]-k_{+5}^{*}\left[\mathrm{SR}_{c}\right]\left[\mathrm{R}_{5-\mathrm{HT} 4}\right] \\
& \mathrm{d}\left[\mathrm{SR}_{c}-\mathrm{R}_{5-\mathrm{HT} 4}-\mathrm{G}\right] / \mathrm{d} t=k_{+7}\left[\mathrm{SR}_{c}-\mathrm{R}_{5-\mathrm{HT} 4}\right]\left(\left[\mathrm{G}^{0}\right]-\left[\mathrm{SR}_{c}-\mathrm{R}_{5-\mathrm{HT} 4}-\mathrm{G}\right]\right) \\
& -k_{-7}^{*}\left[\mathrm{SR}_{c}-\mathrm{R}_{5-\mathrm{HT} 4}-\mathrm{G}\right] \\
& \mathrm{d}[\mathrm{PhC}] / \mathrm{d} t=k_{+10}^{*}\left[\mathrm{SR}_{c}-\mathrm{R}_{5-\mathrm{HT} 4}-\mathrm{G}\right]\left([\mathrm{PhC}]_{\text {inact }}-[\mathrm{PhC}]\right)-k_{+15}^{*}[\mathrm{PhC}] \\
& \mathrm{d}\left[\mathrm{PIP}_{3}\right] / \mathrm{d} t=-\left(k_{+11}^{*}+k_{+12}^{*}\right)\left[\mathrm{PIP}_{3}\right][\mathrm{PhC}] \\
& \mathrm{d}[\mathrm{Dg}] / \mathrm{d} t=k_{+12}^{*}[\mathrm{PhC}]\left[\mathrm{PIP}_{3}\right]-k_{+18}^{*}[\mathrm{Dg}]\left[\mathrm{Ca}^{2+}\right]_{\text {ier }}^{4}\left([\mathrm{PhC}]_{\text {inact }}-[\mathrm{PhC}]\right) \\
& \mathrm{d}\left[\mathrm{IP}_{3}\right] / \mathrm{d} t=k_{+11}^{*}\left[\mathrm{PIP}_{3}\right][\mathrm{PhC}]-k_{+17}^{*}\left[\mathrm{IP}_{3}\right]\left[\mathrm{R}_{\mathrm{ER}}\right]+\left(k_{-17}^{*}-k_{-19}^{*}\right)\left[\mathrm{IP}_{3}-\mathrm{R}_{\mathrm{ER}}\right] \\
& {\left[R_{\mathrm{ER}}\right]=c_{2}\left[\mathrm{IP}_{3}\right]} \\
& \mathrm{d}\left[\mathrm{IP}_{3}-R_{\mathrm{ER}}\right] / \mathrm{d} t=b_{5}\left[\mathrm{IP}_{3}-\mathrm{R}_{\mathrm{ER}}-\mathrm{Ca}^{2+}\right]-\left(a_{2}+a_{5}\right)\left[\mathrm{Ca}^{2+}\right]_{\text {ier }}\left[\mathrm{IP}_{3}-\mathrm{R}_{\mathrm{ER}}\right] \\
& \mathrm{d}\left[\mathrm{Ca}^{2+}\right]_{\text {ier }} / \mathrm{d} t=\tau^{-1}\left(V_{0}-c_{1} V_{1}\left[\mathrm{IP}_{3}-\mathrm{R}_{\mathrm{ER}}-\mathrm{Ca}^{2+}\right]^{3}\left[\mathrm{Ca}^{2+}\right]_{\text {ier }}-\left[\mathrm{Ca}^{2+}\right]_{\mathrm{ER}}\right) \\
& \left.-V_{3}\left[\mathrm{Ca}^{2+}\right]_{\text {ier }}^{2} /\left(\left[\mathrm{Ca}^{2+}\right]_{\text {ier }}^{2}-k_{+13}^{* 2}\right)\right) \\
& c_{0}=\left[\mathrm{Ca}^{2+}\right]_{\mathrm{ier}}+c_{1}\left[\mathrm{Ca}^{2+}\right]_{\mathrm{ER}} \\
& \mathrm{d}[\mathrm{PkC}] / \mathrm{d} t=k_{+8}^{*}[\mathrm{Dg}]\left[\mathrm{Ca}^{2+}\right]_{\text {ier }}^{4}\left([\mathrm{PhC}]_{\text {inact }}-[\mathrm{PhC}]\right)-k_{-8}^{*}[\mathrm{PkC}] \\
& \mathrm{d}[\operatorname{Pr}]_{p} / \mathrm{d} t=k_{+9}^{*}[\mathrm{PkC}]\left([\mathrm{Pr}]-[\mathrm{Pr}]_{p}\right)-k_{-9}^{*}[\mathrm{PPhos}][\mathrm{Pr}]_{p} \\
& {[\mathrm{MAO}]=\left[\mathrm{MAO}^{0}\right]-\left[\mathrm{SR}_{c}-\mathrm{MAO}\right]} \\
& {[\mathrm{G}]=\left[\mathrm{G}^{0}\right]-\left[\mathrm{SR}_{c}-\mathrm{R}_{5-\mathrm{HT} 4}-\mathrm{G}\right] \text {. }}
\end{aligned}
$$

Here: $k^{*}$ are the velocity constants of backward (-) and forward (+) chemical reactions; $k_{v}$ is the diffusion constant; $c_{(i)}, b_{(i)}, V_{0}, \mathrm{~V}_{l}$ are parameters related to the activation of the release of endoplasmic $\mathrm{Ca}^{2+} ; \mathrm{SR}_{\mathrm{v}}$ is vesicular substance $P ; \mathrm{SR}_{\mathrm{f}}$ is the free fraction of serotonin; $\mathrm{SR}_{\mathrm{c}}$ is the SR content in the cleft; MAO is monoamine oxidase; $\mathrm{SR}_{\mathrm{c}}-\mathrm{MAO}$ is serotonin-enzyme complex; $\mathrm{R}_{5-\mathrm{HT} 4}$ is serotonin type 4 receptors; $\mathrm{SR}_{\mathrm{c}}-\mathrm{R}_{5-\mathrm{HT} 4}$ is serotonin-receptor complex; $\mathrm{G}$ is guanine-nucleotide $\mathrm{G}$ protein; $\mathrm{PhC}$ is phospholipase $\mathrm{C} ; \mathrm{PIP}_{3}$ is phosphatidyl inositol 1,4,5triphosphate; $\mathrm{IP}_{3}$ is inositol 1,4,5-triphosphate; $\mathrm{Dg}$ is diacylglycerol; $\mathrm{R}_{\mathrm{ER}}$ is $\mathrm{IP}_{3}$ receptor on the endoplasmic reticulum; $\mathrm{Ca}_{\mathrm{ier}}^{2+}$ is calcium of the endoplasm; $\mathrm{Ca}_{\mathrm{ER}}^{2+}$ is the total amount of calcium stored in the endoplasmic reticulum; $\mathrm{PkC}$ is protein kinase $\mathrm{C}$; $\mathrm{Pr}$ is intracellular protein; PPhos is the protein-phosphatase. 
The system of kinetic equations of the cycle of chemical transformations of serotonin and the activation of serotonin type 3 receptors is:

$$
\begin{aligned}
& \mathrm{d}\left[\mathrm{SR}_{\mathrm{v}}\right] / \mathrm{d} t=-1 / 2 k_{c}^{* *}\left[\mathrm{Ca}^{2+}\right] \varepsilon(t)\left[\mathrm{SR}_{\mathrm{v}}\right] \\
& \mathrm{d}\left[\mathrm{SR}_{\mathrm{f}}\right] / \mathrm{d} t=k_{c}^{* *}\left[\mathrm{Ca}^{2+}\right]\left[\mathrm{SR}_{\mathrm{v}}\right]-k_{d}^{* * *}\left[\mathrm{SR}_{\mathrm{f}}\right]+k_{+6}^{* *}[\mathrm{~S}] \\
& \mathrm{d}\left[\mathrm{SR}_{c}\right] / \mathrm{d} t=k_{\mathrm{d}}^{* *}\left[\mathrm{SR}_{f}\right]-k_{+p}^{* *}\left[\mathrm{SR}_{c}\right] \\
& \mathrm{d}\left[\mathrm{SR}_{p}\right] / \mathrm{d} t=k_{+p}^{* *}\left[\mathrm{SR}_{c}\right]-\left[\mathrm{SR}_{p}\right]\left(k_{+1}^{* *}\left[R_{5-\mathrm{HT} 3}^{0}\right]+k_{+2}^{* *}\left[\mathrm{MAO}^{0}\right]\right) \\
& +\left[\mathrm{SR}_{p}\right]\left(k_{+1}^{* *}\left[\mathrm{SR}-\mathrm{R}_{5-\mathrm{HT} 3}\right]+k_{+2}^{* *}[\mathrm{SR}-\mathrm{MAO}]\right) \\
& +k_{-1}^{* *}\left[\mathrm{SR}-\mathrm{R}_{5-\mathrm{HT} 3}\right]+k_{-2}^{* *}[\mathrm{SR}-\mathrm{MAO}] \\
& \mathrm{d}[\mathrm{SR}-\mathrm{R}] / \mathrm{d} t=k_{+1}^{* *}\left[\mathrm{SR}_{p}\right]\left[\mathrm{R}_{5-\mathrm{HT} 3}^{0}\right]-\left[\mathrm{SR}-\mathrm{R}_{5-\mathrm{HT} 3}\right]\left(k_{-1}^{* *}+k_{+4}^{* *}+k_{+1}^{* *}\left[\mathrm{SR}_{p}\right]\right) \\
& \mathrm{d}[\mathrm{SR}-\mathrm{MAO}] / \mathrm{d} t=k_{+2}^{* *}\left[\mathrm{MAO}^{0}\right]\left[\mathrm{SR}_{p}\right]-[\mathrm{SR}-\mathrm{MAO}]\left(k_{-2}^{* *}+k_{+3}^{* *}+k_{+2}^{* *}\left[\mathrm{SR}_{p}\right]\right) \\
& \mathrm{d}[\mathrm{S}] / \mathrm{d} t=k_{+4}^{* *}\left[\mathrm{SR}-\mathrm{R}_{5-\mathrm{HT} 3}\right]-k_{+6}^{* *}[\mathrm{~S}]+k_{+3}^{* *}[\mathrm{SR}-\mathrm{MAO}] \\
& {\left[\mathrm{R}_{5-\mathrm{HT} 3}\right]=\left[\mathrm{R}_{5-\mathrm{HT} 3}^{0}\right]-\left[\mathrm{SR}-\mathrm{R}_{5-\mathrm{HT} 3}\right]} \\
& {[\mathrm{MAO}]=\left[\mathrm{MAO}^{0}\right]-[\mathrm{SR}-\mathrm{MAO}]}
\end{aligned}
$$

where: $k_{-,}+$are the constants of backward and forward chemical reactions, $\mathrm{k}_{\mathrm{c}}$ is the affinity constant, $k_{\mathrm{d}}$ is the constant of diffusion, $k_{+p}$ is the velocity of diffusion of SR on the subsynaptic membrane, $\mathrm{SR}_{\mathrm{v}}$ is vesicular serotonin and its free $\left(\mathrm{SR}_{\mathrm{f}}\right)$ and postsynaptic $\left(\mathrm{SR}_{p}\right)$ fractions, $\mathrm{R}_{5-\mathrm{HT} 3}$ is serotonin type 3 receptors, $\mathrm{SR}-\mathrm{R}_{5-\mathrm{HT} 3}$ is serotonin-receptor complex, $\mathrm{SR}$ $\mathrm{MAO}$ is serotonin-enzyme complex; $\mathrm{S}$ is products of chemical reactions.

The generation of EPSP potentials

$$
C_{p} \frac{\mathrm{d} \varphi_{p}^{+}}{\mathrm{d} t}+\varphi_{p}^{+}\left(-\Omega[\cdots]+\mathrm{R}_{\mathrm{v}}^{-1}\right)=\varphi_{\mathrm{R}}^{+} / \mathrm{R}_{\mathrm{v}}, \quad[\cdots]=\left\{\begin{array}{l}
{[\mathrm{ACh}-\mathrm{R}]} \\
{\left[\mathrm{SR}-\mathrm{R}_{5-\mathrm{HT} 3}\right]} \\
{\left[\mathrm{SR}-\mathrm{R}_{5-\mathrm{HT} 4}\right]}
\end{array}\right.
$$

where $C_{p}$ is the capacitance of the subsynaptic membrane, $\Omega$ is the empirical constant, $\mathrm{R}_{\mathrm{v}}$ is the general resistance of the extra synaptic structures, and $\varphi_{R}^{+}$is the resting potential.

The kinematic equation of the motion of the bolus along the tube is governed by:

$$
\eta \frac{\mathrm{d} Z_{c}}{\mathrm{~d} t}+F_{d}=\int_{z_{1}}^{z_{2}} \int_{r_{0}}^{r} F_{c} \mathrm{~d} z \mathrm{~d} \zeta
$$

where: $F_{c}, F_{d}$ are the contact force and the force of dry friction, respectively, and $\eta$ is the coefficient of viscous friction. During all stage of dynamic reaction the points of the wall lie over the surface of the sphere:

$$
\mathfrak{\Re}_{c}=\left\{\left(Z_{c}-u_{z}\right)^{2}+\left(r_{0}+u_{r}\right)^{2}+\left(r_{0}+u_{s}\right)^{2}-R^{2}<0\right\}, \quad z \in\left[z_{1}, z_{2}\right]
$$

where: $\mathbf{u}=\left(u_{z}, u_{r}, u_{s}\right)$ is the displacement vector; $Z_{c}$ is the position of the center of the sphere at time $t ; R$ is the maximal radius of the sphere; and $z_{1}, z_{2}$ are the boundary points of contact of the sphere and the wall. 
The initial conditions assume that the system is in the resting state and the position of the center of the bolus is given:

$$
t=0: \quad v_{r}=v_{s}=v_{z}=0 ; \quad \varphi_{l}=\varphi_{c}=0 ; \quad \varphi^{*}=0 ; \quad \varphi_{p}^{+(-)}=0 ; \quad Z_{c}=Z_{c}^{0} .
$$

We assume that the oral (left) and aboral (right) ends of the bioshell are rigidly fixed throughout:

$$
t>0: r\left(\tilde{s}_{1}, 0\right)=r\left(\tilde{s}_{1}, l\right)=r_{0}, \quad s\left(\tilde{s}_{1}, 0\right)=s\left(\tilde{s}_{1}, l\right)=0, \quad z\left(\tilde{s}_{1}, 0\right)=0, \quad z\left(\tilde{s}_{1}, l\right)=l .
$$

The following sequences of physiological events have been modeled:

i) excitation/discharge of ICC by an input signal of intensity $\varphi_{0(i)}$ and duration $t_{0}$ with the generation of the action potential, $\varphi_{(i)}$ :

$$
\varphi_{i}= \begin{cases}\varphi_{0(i)}(t), & 0<t<t_{0} \\ 0, & t \geq t_{0}\end{cases}
$$

ii) initiation of electrochemical processes of nerve-pulse transmission at the cholinergic synapse located at $\left(\tilde{s}_{1}=0, \tilde{s}_{2}=0\right)$ and generation of fEPSPs: $\varphi_{p(l)}^{+}, \varphi_{p(c)}^{+}$, on the circular and longitudinal smooth muscle layers;

iii) generation of the waves of depolarization, $\varphi_{l}$ and $\varphi_{c}$ :

$$
\varphi_{l}\left(\tilde{s}_{1}\right)=\left\{\begin{array}{ll}
\varphi_{p(l)}^{+}(t), & 0<t<t_{0(l)} \\
0, & t \geq t_{0(l)}
\end{array} \quad \varphi_{c}\left(\tilde{s}_{1}\right)= \begin{cases}\varphi_{p(c)}^{+}(t), & 0<t<t_{0(c)} \\
0, & t \geq t_{0(c)}\end{cases}\right.
$$

$t_{0(l)}, t_{0(c)}$ is the time of excitation; the right end of the functional unit remains unexcited throughout:

$$
\varphi_{l}\left(\tilde{s}_{2}\right)=0, \varphi_{c}\left(\tilde{s}_{2}\right)=0(t \geq 0)
$$

iv) as the level of depolarization, $\varphi_{l}$ and $\varphi_{c}$, exceeds the threshold values $\phi_{l}^{*}=30 \mathrm{mV}$, $\phi_{c}^{*}=30 \mathrm{mV}$, spatially distributed oscillators become connected and there is an activation of $L$-type $\mathrm{Ca}^{2+}$-channels on the smooth muscle membrane $\varphi(t)=\varphi_{l}(t)$, $\varphi(t)=\varphi_{c}(t)$, otherwise $\varphi(t)=0$

v) increased influx of $\mathrm{Ca}^{2+}$ ions, development of forces of contraction-relaxation, $T_{c, l}$, deformation of the wall of the organ, and change of intraluminal pressure, $p$; and

vi) generation of contact forces along the surface of the non-deformable solid bolus and its propulsion.

The effects of 5-HT and ACh alone on bolus propulsion by the functional unit were studies in each numerical experiment and as a result of the interaction of 5-HT and ACh through their conjoint activation of different receptor types. The effect of drugs is achieved by varying conductances for affected channels or parameters involved in the second messenger system pathway. The model presented here contains a large number of empirical parameters and constants. Their values could be obtained from Ref. [21] or at (http://com2mac.postech.ac.kr/ Lecture/Lec-15.pdf). 


\section{Numerical algorithm}

Let $\bar{\omega}_{h}=\left\{\left(s_{1, i}, s_{2, j}\right)=\left(i h_{1}, j h_{2}\right), \quad i=\overline{0, N_{1}}, j=\overline{0, N_{2}}, N_{1} h_{1}=2 \pi R_{0}, N_{2} h_{2}=1\right\}$ be the uniform mesh on the cylinder $S$ and let $\gamma=\bar{\omega}_{h} \cap \partial S$ be the boundary. Assume $\omega_{\tau}=$ $\{j \tau, j=0,1, \ldots ; \tau>0\}$ is the temporal mesh in $R_{1}^{+}=\left\{t \in R^{l}: t>0\right\}$. The unknown functions, i.e. velocities, displacements, etc. are defined at the points $\omega_{h} \times \omega_{\tau}$. The myoelectrical pools are located at the subregions $\mathrm{K}_{h}=\mathrm{K}_{h, i, j}=\left\{\left(s_{1, i}, s_{1, i+1}\right) \times\left(s_{2, j}, s_{2, j+1}\right)\right\}$.

The additive finite-difference scheme of alternating directions is adopted to solve (10):

$$
\begin{gathered}
C_{m} \frac{\varphi_{h}^{j+1 / 2}-\varphi_{h}^{j}}{\tau}-0.5 \bar{I}_{m 1, h}\left(\nabla_{h, 1}^{2} \varphi_{h}^{j+1 / 2}+\nabla_{h, 2}^{2} \varphi_{h}^{j}\right)+I_{h, \text { ionic }}^{*}\left(\varphi_{h}^{j+1 / 2}\right)=0, \\
C_{m} \frac{\varphi_{h}^{j+1}-\varphi_{h}^{j+1 / 2}}{\tau}-0.5\left(\nabla_{h, 1}^{2} \varphi_{h}^{j+1 / 2}+\nabla_{h, 2}^{2} \varphi_{h}^{j+1}\right)=I_{m 2, h}\left(\varphi_{h}^{j+1 / 2}\right) .
\end{gathered}
$$

Here,

$$
\begin{aligned}
& \varphi_{h}^{\beta}=\left.\varphi_{h}\right|_{t=\beta \tau}, \quad \nabla_{h, \alpha}^{2} \varphi=\left(g_{0 s_{\alpha}}^{*} / \lambda_{h, \alpha}\right) \varphi_{\bar{s}_{\alpha} s_{\alpha}} \alpha=1,2 ; \\
& \varphi_{\bar{s}_{\alpha} s_{\alpha}}=\left\{\varphi\left(s+h_{\alpha} \mathrm{e}_{\alpha}^{0}\right)-2 \varphi(s)+\varphi\left(s-h_{\alpha} \mathrm{e}_{\alpha}^{0}\right)\right\} / h_{\alpha}^{2}, \quad \alpha=1,2
\end{aligned}
$$

are finite-difference spatial derivatives in the directions $s_{\alpha}, \mathrm{e}_{\alpha}^{0}$ is a unit vector along the $s_{\alpha}$ axis, and $\lambda_{h \alpha}$ are mesh-point approximations of the rates of elongation $\lambda_{c}$ or $\lambda_{l}$, respectively, $\tilde{I}_{m 1, h}$ and $\tilde{I}_{m 1, h}\left(\varphi_{h}^{j+1 / 2}\right)$ are approximate functions $I_{m 1}, I_{m 2}(\varphi)$ on the temporal level $t+\tau / 2$. They are calculated using the bilinear interpolation of $\nabla_{h, \alpha}^{2} \varphi_{h}^{j+1 / 2}$ on a finite element $\mathrm{K}_{h}$. The Chebyshev cubature formulae with $3 \times 3$-axis are employed to calculate the integrals on $\mathrm{K}_{h}$, $I_{h, \text { ionic }}^{*}$ is calculated using the Euler implicit scheme.

The following iterative method is used to solve equation (15):

$$
C_{m} \frac{{ }^{k+1}{ }_{h}^{j+1 / 2}-\varphi_{h}^{j}}{\tau}-0.5 I_{m 1, h}\left(\nabla_{h, 1}^{2}{ }^{k+1} \varphi_{h}^{j+1 / 2}+\nabla_{h, 2}^{2} \varphi_{h}^{j}\right)+I_{h, \text { ionic }}^{*}\left(\varphi_{h}^{j+1 / 2}\right)=0
$$

where $k=0,1, \ldots, \varphi_{h}^{j+1 / 2}$.

The vectors $\mathbf{v}=\left(v_{r}, v_{s}, v_{z}\right)$, and $\mathbf{u}=(r, s, z)$ are calculated from:

$$
\begin{aligned}
& \left\{\begin{array}{l}
\gamma_{0} \mathbf{v}_{h, t}(t)+R \tilde{\lambda}(\mathbf{u})_{t}(t)+\Lambda\left(\hat{\mathbf{v}}_{h}, \hat{\mathbf{u}}_{h}\right)(t+\tau)=0, \\
\mathbf{u}_{h, t}(t)=\mathbf{v}_{h}(t+\tau), \quad t \in \omega_{\tau},
\end{array}\right. \\
& \left.\mathbf{u}_{h}\right|_{\gamma}=0,\left.\quad \mathbf{u}_{h}\right|_{t=0}=u_{0},\left.\quad \mathbf{v}_{h}\right|_{t=0}=0 .
\end{aligned}
$$

Here: $\tilde{\lambda}=\left(\lambda_{h, c}, \lambda_{h, l}\right), \eta_{t}(t)=[\eta(t+\tau)-\eta(t)] / \tau$ are finite approximations of the vector $\left(\lambda_{c}, \lambda_{l}\right)$ and finite difference partial derivative with respect to $t$, respectively. The operators $R$ and $\Lambda$ are approximations of corresponding parts of the spatial operator. The finite element method with bilinear finite elements is used to build $R, \Lambda . \Lambda_{\hat{\Omega}_{c}, \varepsilon}$ is the approximation of the 
penalty operator for the $\Omega_{c}$ and is defined as:

$$
\begin{gathered}
\left(\Lambda_{\mathfrak{S}_{c}}\left(\mathbf{u}_{h}\right), \boldsymbol{\eta}_{h}\right)=\left(\int_{S} \frac{\max \{0,1-\sigma\}}{\varepsilon} \sqrt{g}\left(n_{r} \eta_{r h}+n_{s} \eta_{s h}+n_{z} \eta_{z h}\right) \mathrm{d} S\right) \\
\forall \boldsymbol{\eta}_{h}=\left(\eta_{r h}, \eta_{s h}, \eta_{z h}\right):\left.\quad \boldsymbol{\eta}_{h}\right|_{\gamma}=0,
\end{gathered}
$$

where: $\sigma=R / \sqrt{r_{h}^{2}+\left(z_{h}-Z_{c}\right)^{2}}, \varepsilon>0$ is the penalty parameter. Integrals of each rectangle $\mathrm{K}_{h}$ in the region $S$ are calculated using the formulae of the central rectangles.

Vectors $\mathbf{u}_{h}(t+\tau), \mathbf{v}_{h}(t+\tau)$ and the position of the bolus, $Z_{c, h}(t+\tau)$, are calculated using the two step iterative process:

i) compute $\stackrel{k+1}{\mathbf{v}}_{h}$ from:

$$
\begin{aligned}
& B \frac{\stackrel{k+1}{\mathbf{V}}{ }_{h}-\mathbf{v}_{h}}{\nu}=-\gamma_{0}^{-1}{ }^{k} \mathbf{r} \\
& \stackrel{k}{\mathbf{r}}=\left(\mathbf{v}_{h}^{k}-\mathbf{v}_{h}\right)(t)+R\left(\vec{\lambda}\left(\mathbf{u}_{h}^{k}\right)-\vec{\lambda}\left(\mathbf{u}_{h}\right)\right)+\tau \Lambda\left(\mathbf{v}_{h}, \mathbf{u}_{h}+\tau_{\mathbf{v}_{h}}^{k}\right)(t) \\
& +\tau \Lambda_{\mathfrak{\aleph}_{c}}\left(\mathbf{u}_{h}+\tau \mathbf{v}_{h}^{k}\right)(t),
\end{aligned}
$$

ii) compute ${ }^{k+1}{ }_{h}$ from:

$$
\stackrel{k+1}{\mathbf{u}}_{h}=\mathbf{u}_{h}+\tau^{k+1} \mathbf{v}_{h} .
$$

iii) compute the bolus center position $Z_{c, h}(t+\tau)$ :

$$
\eta \frac{\stackrel{k+1}{Z}_{c, h}-Z_{c, h}}{\tau}+F_{d}=\stackrel{k}{F}_{c, h}
$$

where:

$$
\left(\stackrel{k}{F}_{c, h}, \eta_{h}\right)=\int_{S_{k}}\left(T_{c} \mathrm{e}_{1 z} \sqrt{g_{22}}+T_{L} \mathrm{e}_{2 z} \sqrt{g_{11}}\right) \operatorname{sign}\left(V_{z}\right) \eta_{h} \mathrm{~d} S
$$

and $S_{k}$ is the contact surface of the shell with the $\Omega_{c}$ on the $k$ th-step of integration. Here, $B=\operatorname{diag}\left(B_{1}, B_{2}, B_{3}\right)$ and $B_{\beta}$ are operators of the form:

$$
B_{\beta}=\left(I+\omega \omega_{k} B_{s_{1}}\right)\left(I+\omega \omega_{k} B_{s_{2}}\right), \quad \beta=1,2,3 .
$$

The operators

$$
B_{s_{\alpha}} \eta=-\eta_{\bar{s}_{\alpha} s_{\alpha}}
$$

act in the specific spaces of the mesh functions. They are defined by the boundary conditions (Dirichlet for $B_{s_{2}}$ and periodic for $B_{s_{1}}$ ), $\omega_{k}=\max \left\{k_{c}, k_{l}\right\}$, and $\omega>0, v>0$ are the parameters of iteration, $I$ is the identity matrix. 
For $k=0,1, \ldots$, and sufficiently small $\varepsilon>0$, the steps (i), (ii) are repeated until the following condition is satisfied:

$$
\max \left\{\left\|{ }^{k+1} \mathbf{v}_{h}-\mathbf{v}_{h}\right\|,\left\|\mathbf{u}_{h}-\mathbf{u}_{h}\right\|\right\} \leq \varepsilon \max \left\{\left\|{ }^{k+1} \mathbf{v}_{h}\right\|,\left\|\mathbf{u}_{h}\right\|\right\} .
$$

The finite difference schemes have second order of accuracy with respect to spatial and time variables.

\section{Results}

Results of numerical simulations are presented below. In the resting state, a segment of the gut maintains its initial cylindrical form under the effect of an intraluminal pressure of 1.25 $\mathrm{Pa}$ and an enclosed rigid body. Recordings of electrical activity from the longitudinal and circular smooth muscle layers demonstrate slow wave oscillations in the membrane potential of a constant amplitude $20-25 \mathrm{mV}$ and a frequency $0.5 \mathrm{~Hz}$. There are also concomitant small undulations of active forces in the muscle. The contact forces exerted on the surface of the pellet and the propulsive forces generated by the longitudinal and circle smooth muscle layers are insufficient to propel the bolus along the segment.

\subsection{Simulation of the effect of $n A C h / \mu A C h$ receptors on pellet propulsion}

The discharge of a pacemaker cell initiates the wave of depolarization that propagates at a velocity of $0.05 \mathrm{~cm} / \mathrm{s}$ along the myenteric nervous plexus. The wave reaches the longitudinal smooth muscle syncytium at the neuro-muscular synapses. Here it generates action potentials, $\varphi_{m}=94 \mathrm{mV}$, and a frequency, $\nu=15-25 \mathrm{~Hz}$. As a result, the longitudinal and circular smooth muscle layers generate the total forces of contraction: $T_{l}=89 \mathrm{mN} / \mathrm{cm}$ and $T_{c}=1851 \mathrm{mN} / \mathrm{cm}$, respectively, that are strong enough to move the pellet in the aboral direction. The average velocity of propulsion is $0.8 \mathrm{~cm} / \mathrm{s}$.

\subsection{Simulation of the effect of co-activation of 5-HT, nACh and $\mu A C h$ receptors}

Co-activation of the 5-HT $\mathrm{HT}_{3}$ and nACh receptors affects mainly the dynamics of the spread of excitation within the myenteric nervous plexus. A chaotic pattern of electrical activity with the generation of high amplitude action potentials $(\varphi=89 \mathrm{mV})$ is dominant. The spikes occur randomly at different sites of the functional unit (figure 2(a)). Disrupted strong waves of contraction-relaxation are produced by the longitudinal and circular muscle syncytia, $T_{l}=145 \mathrm{mN} / \mathrm{cm}$ and $T_{c}=1980 \mathrm{mN} / \mathrm{cm}$, that leads to uncoordinated movements of the pellet. The bolus demonstrates "mixing" type of motion, with aboral propulsion at an average velocity of $0.87 \mathrm{~cm} / \mathrm{s}$ (figure $2(\mathrm{~b}),(\mathrm{c})$ ).

\subsection{Simulation of the effects of lotronex (GSK)}

The pharmacological mechanism of action of the drug is a selective 5-HT type 3 receptor antagonism. Under the assumption that there is no ACh in the system, application of Lotronex causes a disruption of the leading front of the depolarization wave and a decrease in the velocity of the spread of excitation within the plexus to $0.04 \mathrm{~cm} / \mathrm{s}$. Lotronex has a prominent 

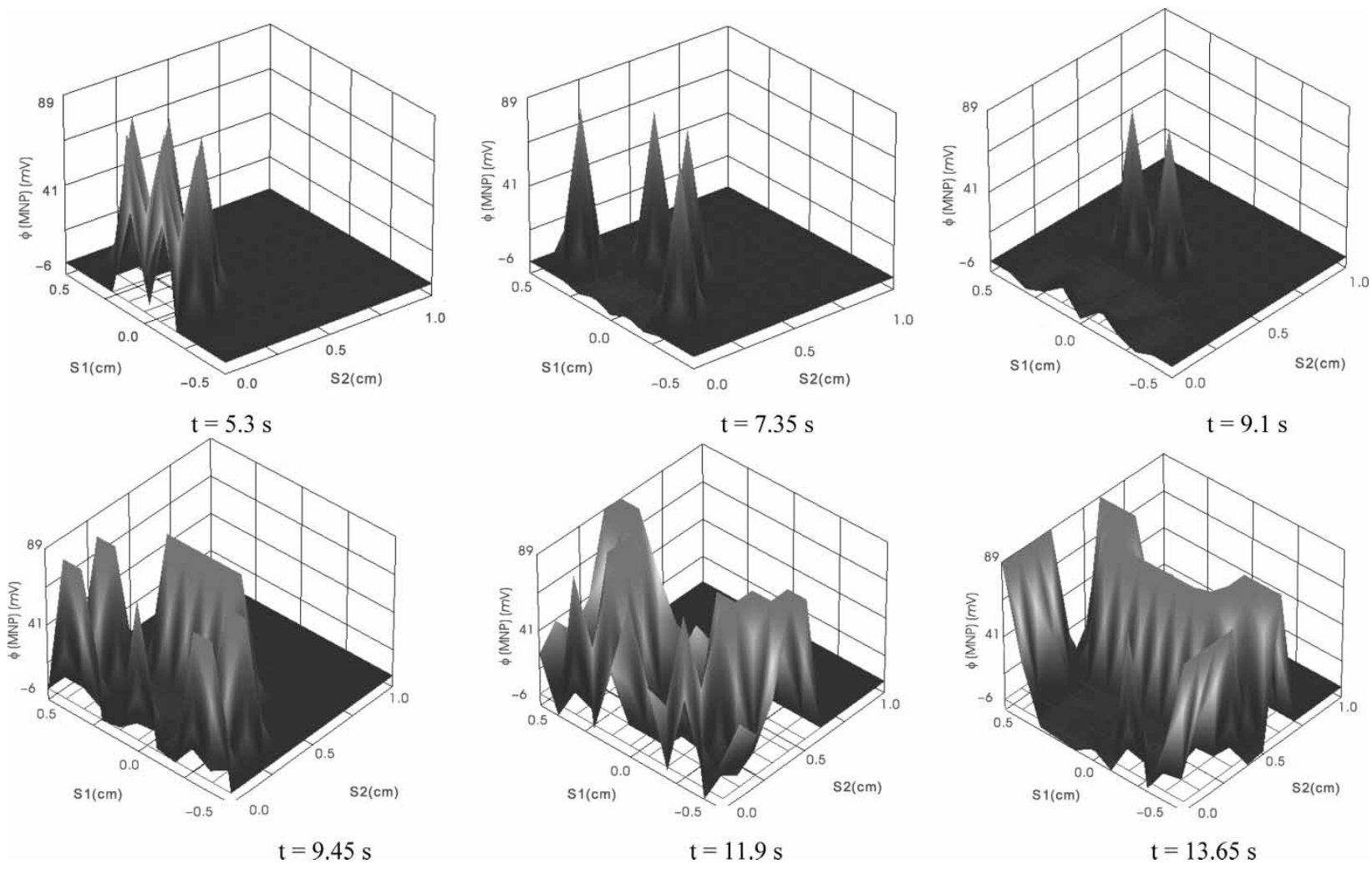

Figure 2. Effects of co-activation of the $5-\mathrm{HT}_{3}$ and $\mathrm{nACh} / \mu \mathrm{ACh}$ receptors on propagation of the wave of depolarization, $\phi_{\mathrm{MNP}}$, along the myenteric nervous plexus (a), development of total forces in the longitudinal, $T_{l}(\mathrm{~b})$ and circular, $T_{c}(\mathrm{c})$ smooth muscle syncytia. The results are presented on open cylindrical envelops where: $s_{1}(\mathrm{~cm})$ and $s_{2}(\mathrm{~cm})$ are spatial coordinates and vertical axes refer to $\phi_{\mathrm{MNP}}(\mathrm{mV}), T_{l}(\mathrm{mN} / \mathrm{cm}) \times 10^{-1}$ and $T_{c}(\mathrm{mN} / \mathrm{cm}) \times 10^{-2}$, respectively. 

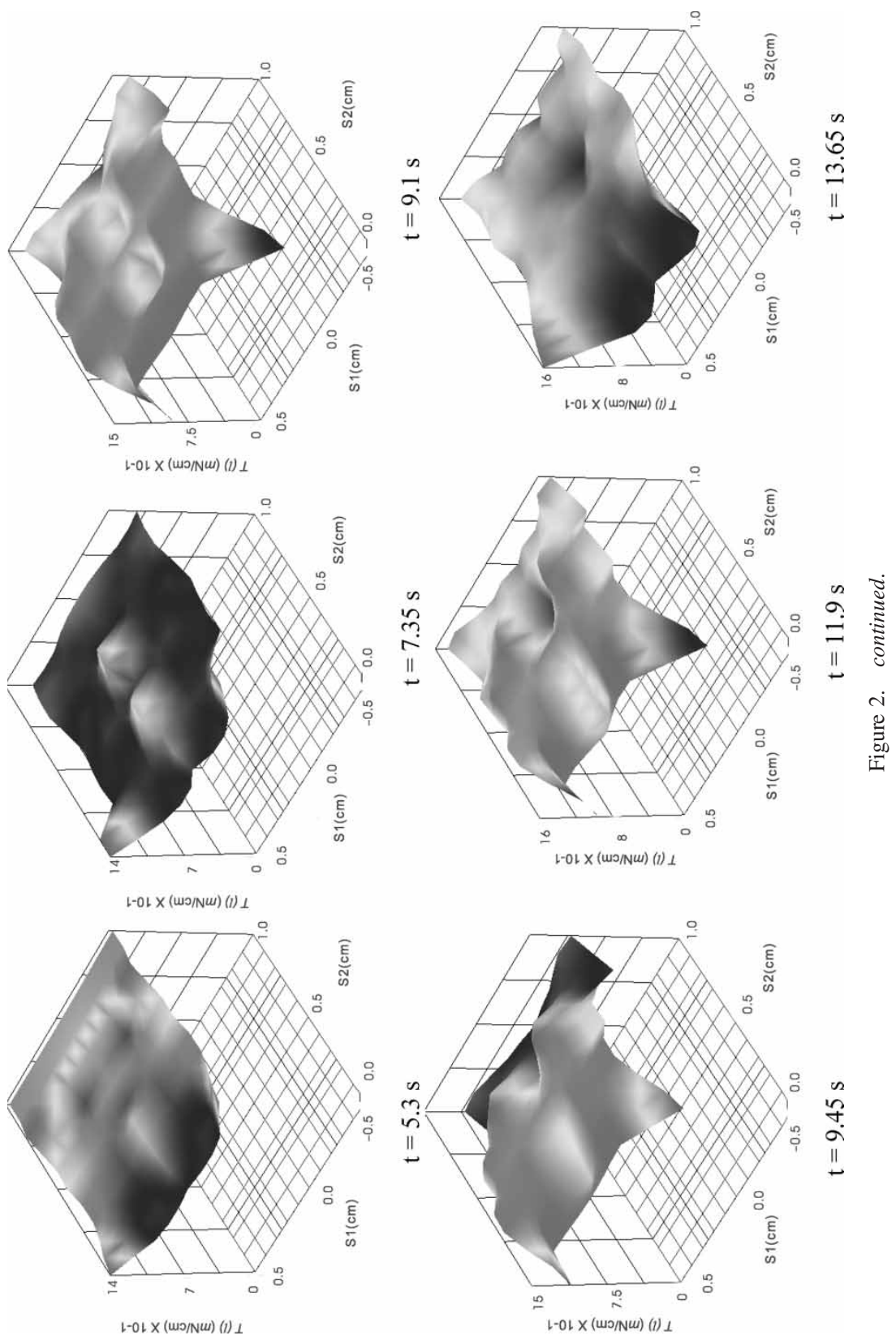

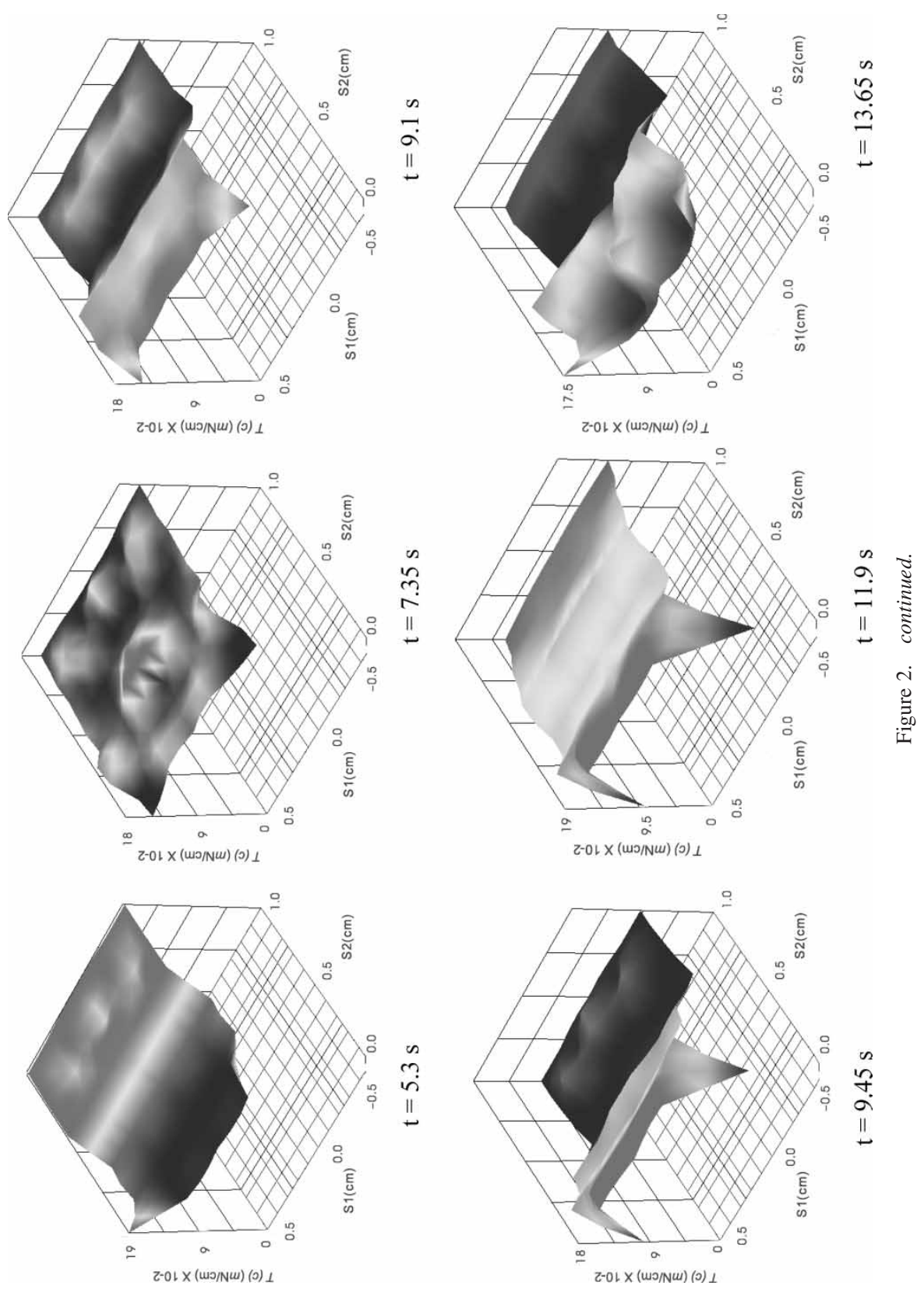
inhibitory affect on the firing rate and the amplitude of action potentials that are recorded on the crests of slow waves (figure 3). However, the frequency of slow waves is increased, $27<t<54$ (s). Smooth muscle syncytia become hyperpolarized with the resting membrane potential: $\varphi_{m \text { (rest) }}=-68.2 \mathrm{mV}$. The above changes in electrical activity cause a significant reduction in the intensity of active force of contraction in the circular, $T_{c}^{a}=1.3 \times 10^{-2} \mathrm{mN} / \mathrm{cm}$, and longitudinal, $T_{l}^{a}=70 \mathrm{mN} / \mathrm{cm}$, smooth muscle layers. As a result there is an impaired propulsive activity of the pellet which moves at a speed of $0.4 \mathrm{~cm} / \mathrm{s}$.

Co-release of ACh at the neuro-neuronal and neuro-muscular junctions at $54<t<126$ (s) with a subsequent activation of the $\mathrm{nACh}$ and $\mu \mathrm{ACh}$ receptors restores normal dynamics of the generation of high amplitude action potentials of average amplitude max $\varphi_{m}=67.4 \mathrm{mV}$. Long-lasting, $72(\mathrm{~s})$, tonic type contractions of $\max T_{c}^{a}=4.5 \times$ $10^{-2} \mathrm{mN} / \mathrm{cm}$ are recorded in the circular layer. Dynamics of the total force distribution in the longitudinal and circular smooth muscle layers along the surface of the functional unit is shown in figure 4. Intense non-propagating contractions of $\max T_{l}^{a}=15 \times 10^{-1} \mathrm{mN} / \mathrm{cm}$ and $\max T_{c}^{a}=3.4 \times 10^{-2} \mathrm{mN} / \mathrm{cm}$ are observed in the vicinity of the right boundary (oral end) of the gut. They significantly impair propulsion of the pellet which comes to a complete stand still. Only after the removal of ACh from the system does the functional unit regains its propulsive activity in presence of Lotronex.

\subsection{Simulation of the effects of selective 5-HT $\mathrm{H}_{4}$ receptor agonists}

Treatment of a segment of the gut with $5 \mathrm{HT}_{4}$ receptor agonists-Zelnorm (Novartis) or TS951, sustains the reciprocal relationship in contraction-relaxation between the longitudinal and circular smooth muscle layers. No significant changes are observed in the dynamics of the propagation of the electrical wave of excitation within the myenteric plexus after application of the drugs. The results show an increase in tone of the longitudinal muscle
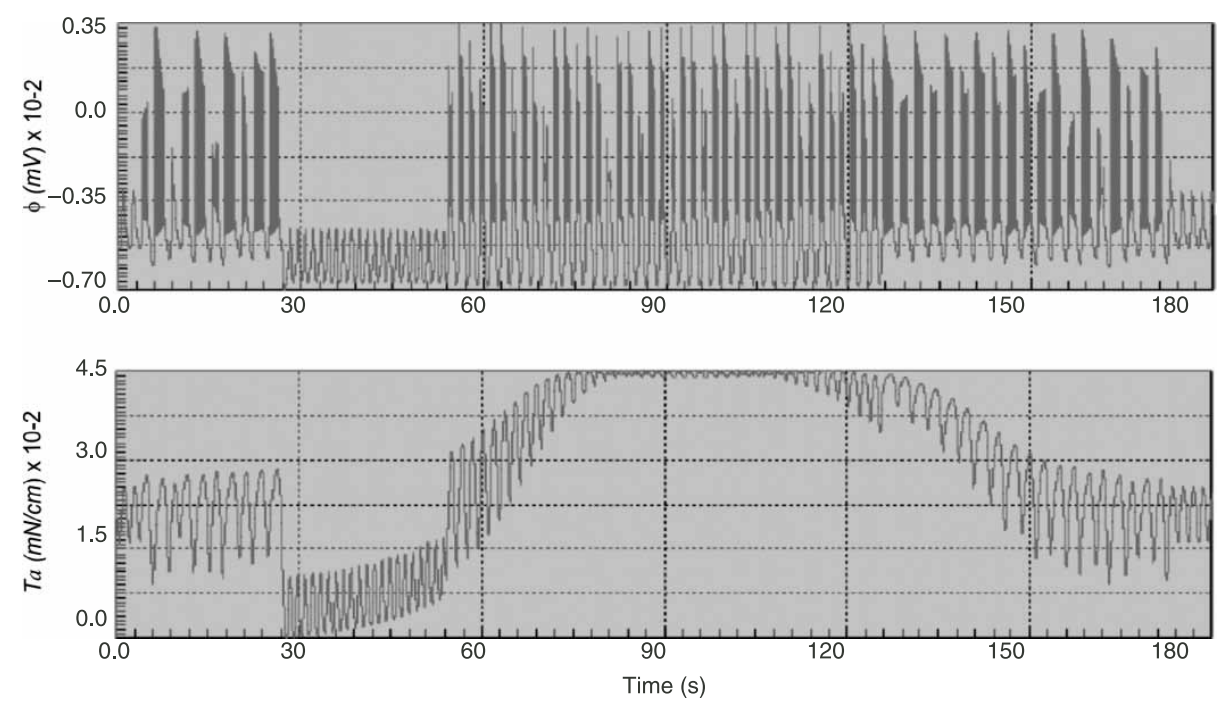

Figure 3. Effects of Lotronex on gut motility. The upper trace shows myoelectrical activity, $\phi_{c}(\mathrm{mV}) \times 10^{-2}$ and the lower trace is the concomitant force of contraction, $T_{c}^{a}(\mathrm{mN} / \mathrm{cm}) \times 10^{-2}$ in the circular smooth muscle layer. 

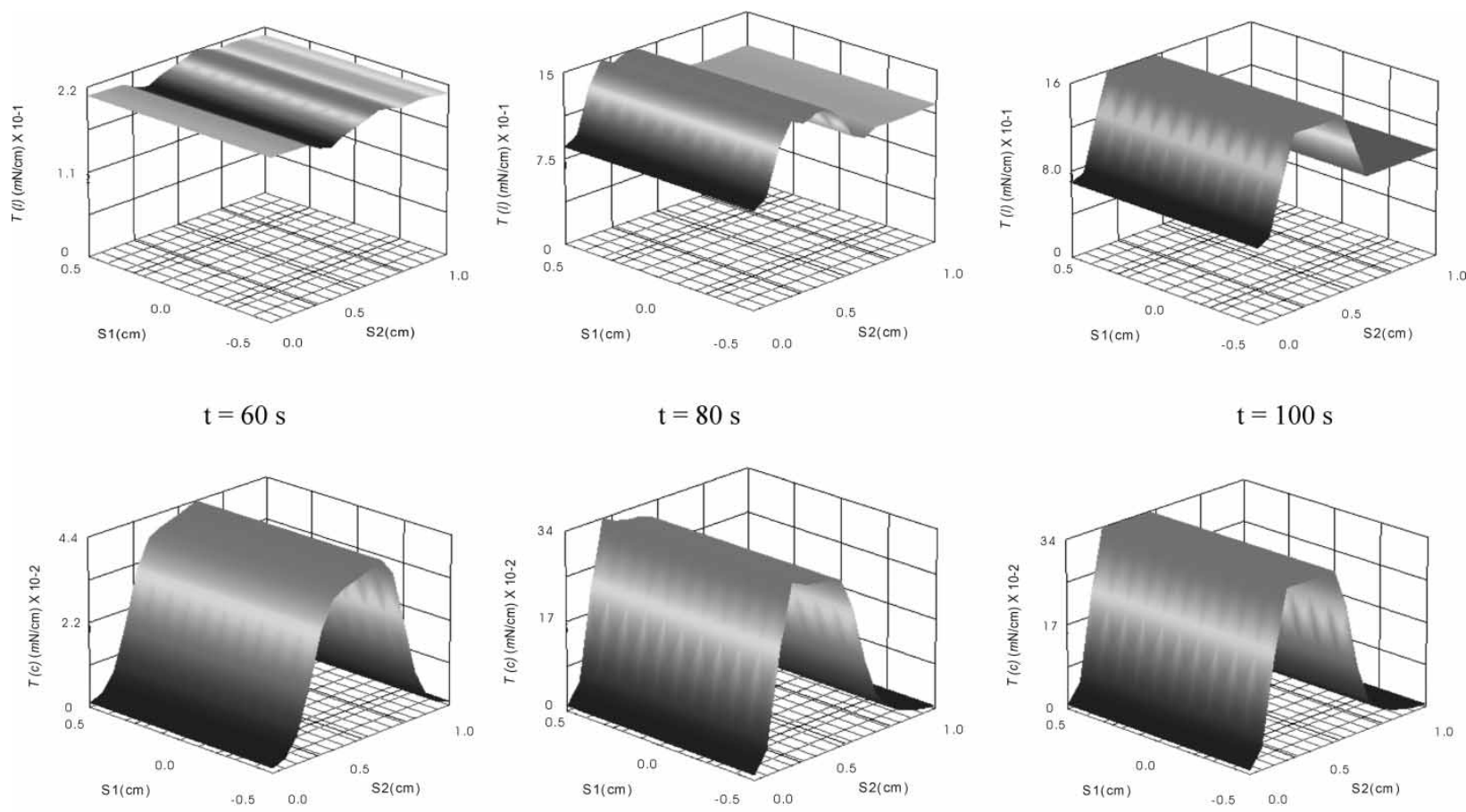

$$
\mathrm{t}=80 \mathrm{~s}
$$

$\mathrm{t}=100 \mathrm{~s}$

Figure 4. Dynamics of the development of total forces in the longitudinal and circular smooth muscle syncytia after co-release of Ach and application of Lotronex. The results are presented on open cylindrical envelops where: $s_{1}(\mathrm{~cm})$ and $s_{2}(\mathrm{~cm})$ are spatial coordinates and vertical axes to $T_{l}(\mathrm{mN} / \mathrm{cm}) \times 10^{-1}$ and $T_{c}(\mathrm{mN} / \mathrm{cm}) \times 10^{-2}$, respectively. 
layer, $T_{l}=205 \mathrm{mN} / \mathrm{cm}$, with no changes in the total force in the inner circular layer. Regular contractions are evenly distributed along the surface of the functional unit and on the contact surface with the pellet. The bolus sustains pure expulsive aboral movement at velocity $1.2 \mathrm{~cm} / \mathrm{s}$ with no mixing being observed.

\subsection{Simulation of the effects of selective $5-\mathrm{HT}_{4}$ receptor antagonists}

Neither GR 113808A nor SDZ205557-selective 5HT 4 receptor antagonists, has any effect on the propagation of the wave of excitation along the myenteric nervous plexus. However, the application of these drugs causes disruption of the migrating myoelectrical complex. Multiple irregular high amplitude $\left(\max \varphi_{m}=54 \mathrm{mV}\right)$ action potentials are recorded from different sites of the syncytia. Although active forces produced by the longitudinal and circular smooth muscle layers are strong enough to initiate and maintain propulsion of the pellet, there is an overall slowdown in movement. The maximal velocity of the pellet is only $0.5 \mathrm{~cm} / \mathrm{s}$.

\subsection{Simulation of effect of Cisapride}

Application of Cisapride results in an increase in excitability of the primary, motor and interneurons of the myenteric nervous plexus. The neurons generate high amplitude and frequency action potentials. The drug does not affect the intensity of the propagation of electrical signals within the myenteric nervous plexus. However, an increase in the frequency of slow waves $(\nu=30 \mathrm{~Hz})$ in the longitudinal and circular smooth muscle syncytia is observed. Long-lasting tonic contractions are induced by both smooth muscle layers with $\max T_{l}=200 \mathrm{mN} / \mathrm{cm}$, and $\max T_{c}=2100 \mathrm{mN} / \mathrm{cm}$. As a result, squeezing of the pellet towards the aboral end, rather than a mixing type of propulsion, is recorded.

\section{Discussion}

With our simulations we were able to study numerically the effects of co-transmission by $\mathrm{ACh}$ and 5-HT on propulsion of a solid non-deformable bolus in the gut. Comparison of the numerical results to in vivo and in vitro experimental data shows satisfactory qualitative and quantitative agreement. Using the isolated guinea pig ileum, Tuladhar et al. [22,23] demonstrated that activation of the $5-\mathrm{HT}_{3}$ receptors by mucosally applied free 5-HT reduces the mechanical threshold for initiation of the peristaltic reflex by 20-26\%. Experimental intracellular recordings from the soma of the primary neurons of the guinea-pig ileum myenteric nervous plexus show that after application of 5-HT [24] the neuron produces long lasting, 2-4 s, trains of action potentials of amplitude $75-80 \mathrm{mV}$ and a frequency ranging from 0.1 to $10(\mathrm{~Hz})$. Our numerical simulation of co-transmission by ACh and 5-HT shows that motor neurons generate high amplitude and frequency action potentials. This response can be attributed to a heteromeric co-assembly of the $5-\mathrm{HT}_{3}$ receptors with nicotinic $\mathrm{ACh}$ ( $\alpha 4$ subunit) receptors [25-28]. Interestingly, application of Cisapride results in a two/three times higher concentration of intracellular $\mathrm{Ca}^{2+}$ and an increase in intensity of total forces than after treatment with natural endogenous serotonin alone [28]. While these experimental findings concur with the results of our simulations, the putative role of the $5-\mathrm{HT}_{3} / \mathrm{nACh}$ ligand-channel co-assembly require further pharmacological investigation. The effects of co-expression of the $5-\mathrm{HT}_{3}, 5-\mathrm{HT}_{4}$ and $\mathrm{nACh}$ receptors on the soma of the $\mathrm{S}$ neuron on the 
dynamics of the electrical signal transduction were analyzed in this study demonstrating a minor role for 5-HT type 3, in contrast to the 5-HT type 4 receptors. The activation of 5- $\mathrm{HT}_{4}$ receptors evoked a long lasting train of fast action potentials. Co-stimulation of (5- $\mathrm{HT}_{3}-$ $\mathrm{nACh})$ and $\left(5-\mathrm{HT}_{4}-\mathrm{nACh}\right)$ receptors has a considerable excitatory effect on neurons resulting in a significant increase in the amplitude and frequency of firing rate. These findings are in line with a possible co-assembly of serotonergic and cholinergic receptors [28-31].

The results indicate that excitation of 5- $\mathrm{HT}_{4}$ receptors only in conjunction with $\mu \mathrm{ACh}$ receptors is able to initiate long-lasting phasic contractions. These findings are supported experimentally by the selective activation of $5-\mathrm{HT}_{4}$ receptors on longitudinal smooth muscle of the large intestine of different species [14,32]. The in vivo and in vitro studies of the effects of Lotronex on contractility and compliance of the colonic muscle demonstrated: (i) the decrease in contractions in the small and large intestines, (ii) the increase in wall compliance of the colon, and (iii) the delay of gut transit time [33-36]. These results support our numerical simulations of pellet propulsion in a segment of the gut. Lotronex causes a decrease in the velocity of propulsion by $50 \%$. Disruption in the wave of depolarization and a delay of the spread of excitation within the myenteric nervous plexus are suggested as mechanisms for the slowdown. Interestingly, co-activation of $\mathrm{nACh}$ and $\mu \mathrm{ACh}$ receptors results in the development of long lasting tonic-type contractions that bring the pellet to a standstill. The loss of reciprocal relationship in contraction-relaxation between the two muscle layers is a possible mechanism.

Selective 5-HT 4 receptor agonists-Zelnorm and/or TS-591, alone do not appear to affect reciprocal relationships in mechanical activity between two smooth muscle layers. Strong contractions in the longitudinal and circular muscle layers cause a rapid expulsion of the pellet with a loss of physiological "mixing". These results concur with previous observations $[7,10,13,26,28,37-39]$ of the effects of TS-591 which demonstrates a significant prokinetic effect on motility of the gut.

The in silico integrative approach enables us to gain insight into the intricate pharmacodynamic and physiological effects of various drugs, which cannot be achieved in neither in vivo or in vitro preparations. This study confirmed numerically that a non-selective strong 5- $\mathrm{HT}_{3}$ and weak $5-\mathrm{HT}_{4}$ - receptor agonist, Cisapride, attains its prokinetic effect through the increase in: (i) influx of $\mathrm{Ca}^{2+}$ ions [28], and (ii) excitability of the primary (AH) and motor (S) neurons that lead to twitch contractions of the smooth muscle syncytium. However, these changes at a cellular level are not correlated linearly with the effects of the drug on the myenteric plexus. Thus, Cisapride does not alter the dynamics of the propagation of electrical signals within the planar neural network formed of AH and S neurons. At the functional unit level, Cisapride causes an increase in frequency of slow waves and fEPSPs which results in the development of tonic-type contractions in the longitudinal and circular smooth muscle layers. The pattern of mechanical propulsive activity changes from "mixing" to the "expulsive" type.

\section{Conclusion}

Comprehensive experimental data on physiology and neuro-pharmacology of the gut, combined with modern computational modeling technology, allows the study, in the "virtual environment", the effects of co-transmission by 5-HT and ACh and receptor polymodality, i.e. $5-\mathrm{HT}_{3}, 5-\mathrm{HT}_{4}, \mathrm{nACh}$ and $\mu \mathrm{ACh}$ type receptors, on bolus propulsion in the gut. To our knowledge this is the first publication that provides numerical simulations of the effects of 
multiple neurotransmission on the dynamics of bolus propulsion. Our numerical investigations suggest the intrinsic mechanisms of co-transmission at the cellular, tissue and organ levels. Such mechanisms cannot be elucidated using existing experimental in vivo or in vitro methods. One advantage of the computer-based approach to study complex biological phenomena is the presentation of findings. The results of our numerical simulations are displayed on cylindrical plane envelopes in contrast to traditional function (membrane potential, force, etc.) vs time presentations. This form of presentation gives the reader a clear three dimensional view of the actual dynamics of physiological processes in a functional unit of the gut rather than a "single electrode" recording at fixed points in space and further interpolation of the results to the whole organ. At this stage it is not possible to provide a convincing comparative evaluation of the numerical results of co-transmission by 5HT and ACh on the dynamics of bolus propulsion: there is not enough experimental data, and further experimental research is needed.

A few words of criticism, however, are raised concerning the model. Although a biomechanical model of the gut as a soft electromyogenic biological shell has been extensively studied and validated physiologically and neuropharmacologically, the model of the intraluminal content as a solid rigid sphere is limited. It could satisfy only certain in vivo or clinical investigations when metallic beads are administered into the gastrointestinal tract to study the transit time. No mathematical models of chyme as a deformable biologically active medium have been proposed until now.

The current model does not include processes of secretion and absorption. These mechanisms undoubtedly have a significant impact on bolus propulsion. It remains a challenging task to formulate such a problem and to solve it. The emphasis would be stress on a numerical solution because high nonlinearities are expected in the problem formulation and these generally preclude analytical solution techniques.

The model allows us to study phenomenologically the effects of different classes of pharmacological compounds on intestinal propulsion and to predict the general trend of their action on the system rather than to provide a detailed quantitative analysis of concentration vs time or drug effect $v$ s time results. This phenomenological approach limits the pharmacological practicality of the model; however, the issue could be resolved in the future.

\section{References}

[1] Buist, M.L., Cheng, L.K., Yassi, R., Bradshaw, L.A., Richards, W.O. and Pullan, A.J., 2004, An anatomical model of the gastric system for producing bioelectric and biomagnetic fileds, Physiological Measurement, 25, 849-861.

[2] Pullan, A.J., Cheng, L., Yassi, R. and Buist, M., 2004, Modelling gastrointestinal bioelectric activity, Progress in Biophysics and Molecular Biology, 85, 523-550.

[3] Kuznetsov, V.L., Troitskaya, V.B., Vershinina, E.A., Polenov, S.A. and Kucher, V.I., 2001, A mathematical model of digestion regulation, Dokl. Akad. Nauk, Doklady Biol. Sci., 138, 224-226.

[4] Tack, J. and Sarnelli, G., 2002, Serotonergic modulation of visceral sensation: upper gastrointestinal tract, Gut, 51, i77-i80.

[5] Galligan, J.J., 1996, Electrophysiological studies of 5-hydrohyryptamine receptors on enteric neurons, Behavioural Brain Research, 73, 199-201.

[6] Zhou, X. and Galligan, J.J., 1999, Synaptic activation and properties of 5-hydro-xytryptamine 3 receptors in myenteric neurons of guinea pig intestine, Journal of Pharmacology and Experimental Therapeutics, 290, $803-810$.

[7] McLean, P.G. and Coupar, I.M., 1996, Stimulation of cyclic AMP formation in the circular smooth muscle of human colon by activation of 5- $\mathrm{HT}_{4}$-like receptors, British Journal of Pharmacology, 117, 238-239.

[8] Johnson, D.S. and Heinemann, S.F., 1995, Detection of 5-HT3R-A, a 5-HT3 receptor subunit, in submucosal an myenteric ganglia of rat small intestine using in situ hybridization, Neuroscience Letters, 184, 7-70. 
[9] Nagakura, Y., Ito, H., Kiso, T., Naitoh, Y. and Miyata, K., 1997, The selective 5-hydroxytryptamine 5-HT $4-$ receptor agonist RS67506 enhances lower intestinal propulsion in mice, The Japanese Journal of Pharmacology, 74, 209-212.

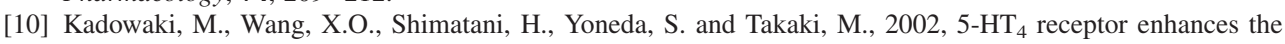
propulsive power of the peristaltic reflex in the rat distal colon, Auton. NeuroSc. Basic \& Clinic., 99, 62-65.

[11] Nagakura, Y., Kamato, T., Nishida, A., Ito, H., Yamano, M. and Miyata, K., 1996, Characterization of 5hydroxytriptamine (5-HT) receptor subtypes influencing colonic motility in conscious dogs, Naunyn-Schmied Archives of Pharmacology, 353, 489-498.

[12] Briejer, M.R., Bosmans, J-P., Van Daele, P., Juzrak, M., Heylen, L., Leysen, J.E., Prins, N.H. and Schuurkers, J.A.J., 2001, The in vivo pharmacological profile of prucalopride, a novel enterokinetic compound, European Journal of Pharmacology, 423, 71-83.

[13] Prins, N.H., Akkermans, L.M.A., Lefebvre, R.A. and Schuurkers, J.A.J., 2000, 5-HT 4 receptors on cholinergic nerves involved in contractility of canine and human large intestine longitudinal muscle, British Journal of Pharmacology, 131, 927-932.

[14] Bush, T.G., Spencer, N.J., Watters, N., Sanders, K.M. and Smith, T.K., 2001, Effects of alosetron on spontaneous migrating motor complexes in murine small and large bowel in vitro, AJP Gastrointestinal and Liver Physiology, 281, G974-G983.

[15] Kajita, S., Ito, C., Kawamura, Y.S., Isobe, Y. and Fukushima, K., 2001, Pharmacological characterization of a novel 5- $\mathrm{HT}_{4}$ receptor agonist, TS-591, in vitro, Pharmacology, 63, 8-16.

[16] Miftakhov, R.N., 1989, Numerical modeling of the small intestine motility. Modern Problems in Biomechanics, Riga, VI, pp. 147-183.

[17] Miftakhov, R.N., Abdusheva, G.R. and Christensen, J., 1999, Numerical simulation of motility patterns of the small bowel. 1. Formulation of a mathematical model, Journal of Theoretical Biology, 197, 89-112.

[18] Miftakhov, R. and Christensen, J., 2001, A physicochemical basis of synaptic transmission in the myenteric nervous plexus. In: R. Poznanski (Ed.) Bioph Neural Networks (New York, NY: Mary Ann Liebert Inc.), pp. $147-176$.

[19] Miftahof, R.N. and Fedotov, E.M., 2004, The concept of the functional unit of the gut. In: M. Rahman, R. Verhoeven and C.A. Brebbia (Eds.) Advances in Fluid Mechanics (Southampton, Boston: WIT Press), pp. 353-361.

[20] Miftahof, R.N. and Fedotov, E.M., 2005, Intestinal propulsion of a solid non-deformable bolus, Journal of Theoretical Biology, 235, 57-70.

[21] Miftahof, R., 2005, Biomechanics of the small intestine, Lecture Note Series, Pohang, Korea, pp. 115

[22] Tuladhar, B.R., Kaisar, M. and Naylor, R.J., 1997, Evidence for a $5 \mathrm{HT}_{3}$ receptor involvement in the facilitation of peristalsis on mucosal application of 5-HT in the guinea pig isolated ileum, British Journal of Pharmacology, 122, 1174-1178.

[23] Tuladhar, B.R., Womack, M.D. and Naylor, R.J., 2000, Pharmacological characterization of the 5-HT receptor mediated contraction in the mouse isolated ileum, British Journal of Pharmacology, 1313, 1716-1722.

[24] Bertrand, P.P., Kunze, W.A.A., Furness, J.B. and Borstein, J.C., 2000, The terminals of myenteric intrinsic primary afferent neurons of the guinea-pig ileum are excited by 5-hydroxytryptamine acting at 5-hydroxytryptamine - 3 receptors, Neuroscience, 101, 459-469.

[25] Holler, C., Freissmuth, M. and Nanoff, C., 1999, G proteins as drug targets, Cellular and Molecular Life Sciences, 55, 257-270.

[26] Van Hooft, J.A., Spier, A.D., Yakel, J.L., Lummis, S.C.R. and Vijverberg, H.P.M., 1998, Promiscuous coassembly of serotonin $5-\mathrm{HT}_{3}$ and nicotinic (4 receptor subunits into $\mathrm{Ca}^{2+}$ - permeable ion channels, Proceedings of the National Academy of Sciences USA, 95, 11456-11461.

[27] Barajas-Lopez, C., Karanjia, R. and Espinosa-Luna, R., 2001, 5-Hydroxytryptamine and atropine inhibit nicotinic receptors in submucosal neurons, European Journal of Pharmacology, 414, 113-123.

[28] Pindon, A., Van Hecke, G., Van Gompel, P., Lesage, A.S., Leusen, J.E. and Jurzak, M., 2002, Differences in Signal transduction of two $5-\mathrm{HT}_{4}$ receptor splice variants: compound specificity and dual coupling with $\mathrm{G} \alpha \mathrm{s}-$ and Gai/o-proteins, Molecular Pharmacology, 61, 85-96.

[29] Legay, C., Saffrey, M.J. and Burnstock, G., 1984, Coexistence of immunoreactive substance P and serotonin in neurons of the gut, Brain Research, 302, 379-382.

[30] Briejer, M.R. and Schuurkers, J.A.J., 1996, 5- $\mathrm{HT}_{3}$ and 5- $\mathrm{HT}_{4}$ receptors and cholinergic and tachykininergic neurotransmission in the guinea-pig proximal colon, European Journal of Pharmacology, 308, 173-180.

[31] Foxx-Orenstein, A.E., Jin, J.G. and Grider, J.R., 1998, 5-HT 4 receptor agonists and $\delta$-opioid receptor antagonists act synergistically to stimulate colonic propulsion, American Journal of Physiology, 275, G979-G983.

[32] Grider, J.R., Foxx-Orstein, A.E. and Jin, I-G., 1998, 5-hydroxytryptamine ${ }_{4}$ receptor agonists initiate the peristaltic reflex in human, rat, and guinea pig intestine, Gastroenterol, 115, 370-380.

[33] Audolfsson, G., Bayguinov, O., Yamamoto, T., Somogyi, G.T., Schraut, W.H., Sanders, K.M. and Bauer, A.J., 1999, Review article: effects of the 5- $\mathrm{HT}_{3}$ receptor antagonist alosetron in neuromuscular transmission in canine and human intestinal muscle, Alimentary Pharmacology and Therapeutics, 13, 39-47. 
[34] Gunput, M.D., 1999, Review article: clinical pharmacology of alosetron, Alimentary Pharmacology and Therapeutics, 13, 70-76.

[35] Humphrey, P.P.A., Bountra, C., Clayton, N. and Kozlowski, K., 1999, Review article: the therapeutic potential of $5-\mathrm{HT}_{3}$ receptor antagonists in the treatment of irritable bowel syndrome, Alimentary Pharmacology and Therapeutics, 13, 31-38.

[36] Lördal, M. and Hölstrom, P.M., 1999, Serotonin stimulates migrating myoelectric complex via 5-HT 3 receptors dependent on cholinergic pathways in rat small intestine, Neurogastroenterol and Motility, 11, 1-10

[37] De Ponti, F., Crema, F., Moro, E., Nardelli, G., Croci, T. and Frigo, G.M., 2001, Intestinal motor stimulation by the 5-HT - receptor agonist ML10302: differential involvement of tachykininergic pathways in the canine small bowel and colon, Neurogastroenterol and Motility, 13, 543-553.

[38] Bharucha, A.E., Camilleri, M., Haydock, S., Ferber, I., Burton, D., Cooper, S., Tompson, D., Fitzpatrick, K., Higgins, R. and Zinsmeister, A.R., 2000, Effects of a serotonin 5-HT 4 receptor antagonist SB-207266 on gastrointestinal motor and sensory function in humans, Gut, 4, 667-674.

[39] Briejer, M.R., Prins, N.H. and Schuurkers, J.A.J., 2001, Effects of the enterokinetic prucalopride (R093877) on colonic motility in fasted dogs, Neurogastroenterol and Moilityt, 13, 465-472. 


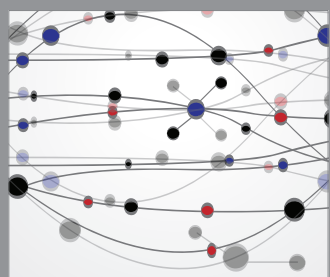

The Scientific World Journal
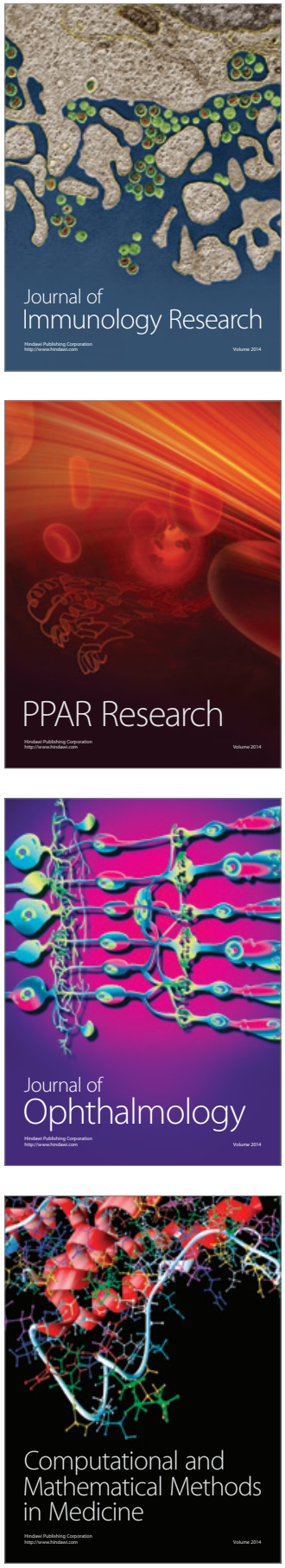

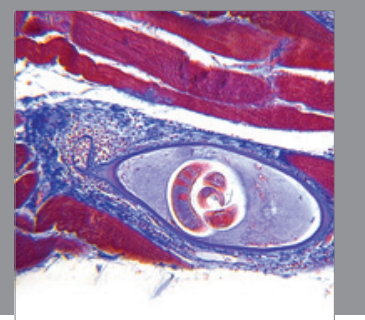

Gastroenterology

Research and Practice
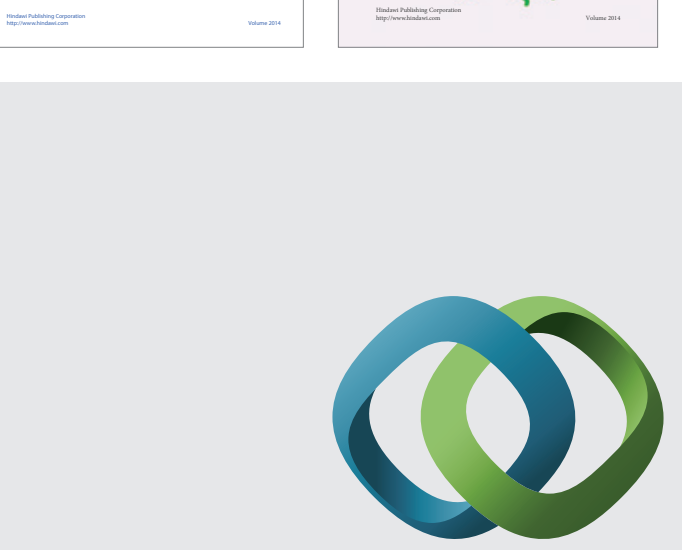

\section{Hindawi}

Submit your manuscripts at

http://www.hindawi.com
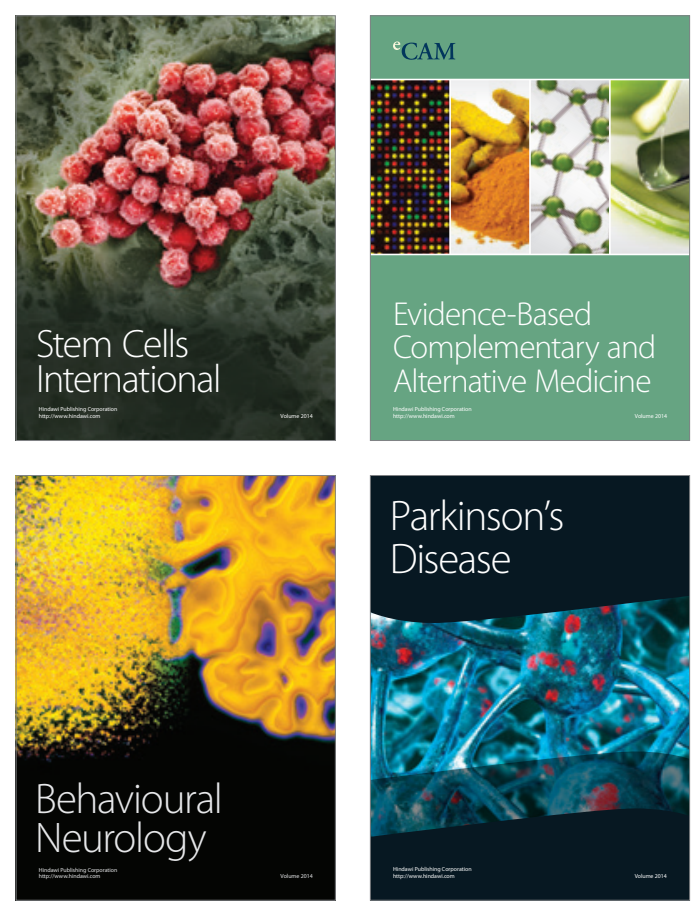

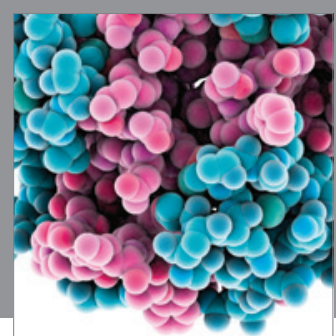

Journal of
Diabetes Research

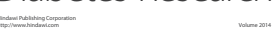

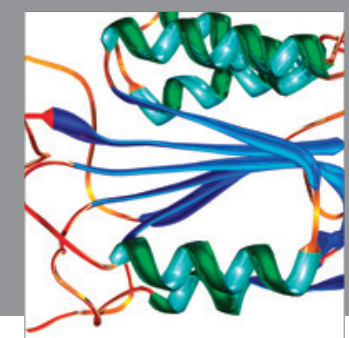

Disease Markers
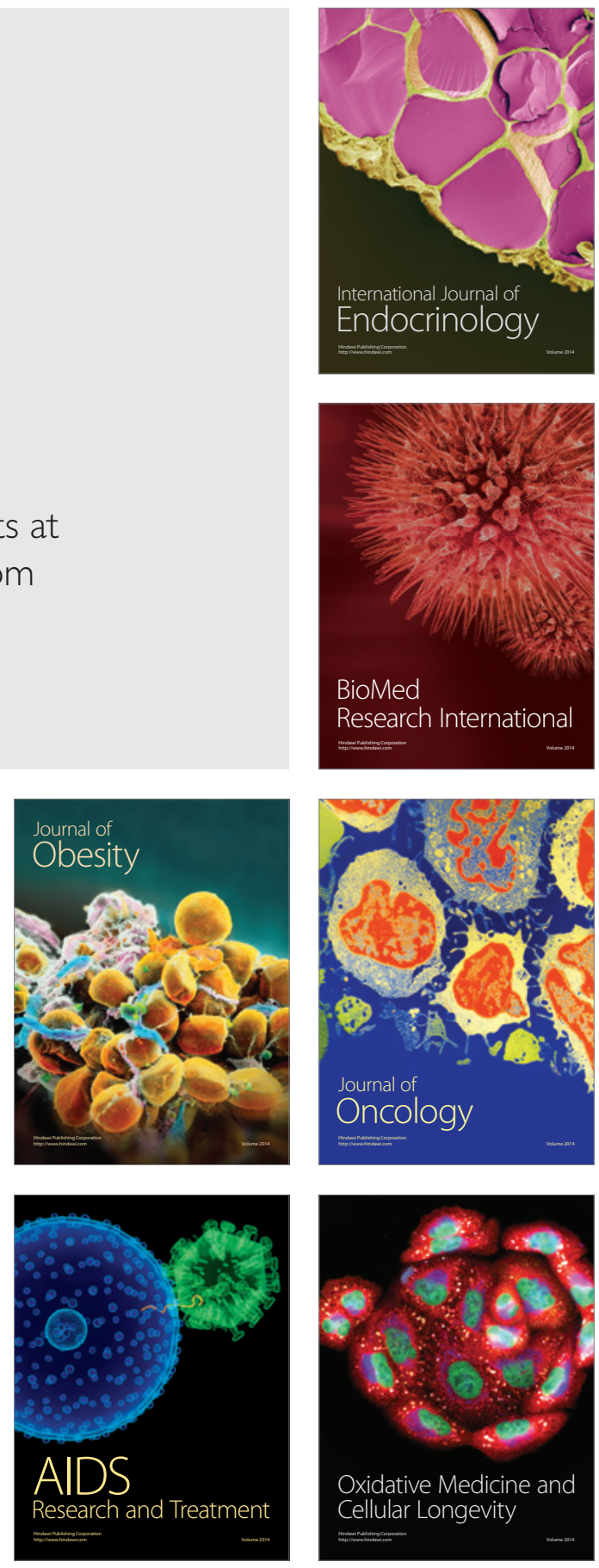